Revue des patrimoines

$21 \mid 2013$

De l'art de bâtir aux champs à la ferme moderne

\title{
La Cité paysanne de Marckolsheim (1940-1944). Étude et protection d'un quartier de fermes modèles en Alsace
}

Clémentine Albertoni, Laurine Sandoval et Séverine Wodli

\section{(2) OpenEdition \\ Journals}

Édition électronique

URL : http://journals.openedition.org/insitu/10500

DOI : 10.4000/insitu. 10500

ISSN : 1630-7305

Éditeur

Ministère de la culture

Référence électronique

Clémentine Albertoni, Laurine Sandoval et Séverine Wodli, « La Cité paysanne de Marckolsheim

(1940-1944). Étude et protection d'un quartier de fermes modèles en Alsace », In Situ [En ligne],

21 | 2013, mis en ligne le 22 juillet 2013, consulté le 01 mai 2019. URL : http://

journals.openedition.org/insitu/10500 ; DOI : 10.4000/insitu.10500

Ce document a été généré automatiquement le 1 mai 2019.

\section{(c) $(1) \Theta \theta$}

In Situ Revues des patrimoines est mis à disposition selon les termes de la licence Creative Commons Attribution - Pas d'Utilisation Commerciale - Pas de Modification 4.0 International. 


\title{
La Cité paysanne de Marckolsheim (1940-1944). Étude et protection d'un quartier de fermes modèles en Alsace
}

\author{
Clémentine Albertoni, Laurine Sandoval et Séverine Wodli
}

La notion de "patrimoine culturel» telle que définie par l'UNESCO élargit considérablement les problématiques traditionnelles de définition, de protection et de valorisation du patrimoine monumental ${ }^{1}$. L'architecture du $\mathrm{XX}^{\mathrm{e}}$ siècle, l'architecture de série, le paysage, le patrimoine rural et agricole qu'il soit matériel ou immatériel, sont pris en compte dans le corpus des édifices « qui ont une valeur universelle exceptionnelle du point de vue de l'histoire, de l'art ou de la science ${ }^{2}$ ». La Direction régionale des affaires culturelles (DRAC) d'Alsace a choisi de tenir compte des spécificités de l'histoire régionale dans la définition de sa politique patrimoniale. À ce titre, la Conservation régionale des monuments historiques $(\mathrm{CRMH})$ étudie et protège des monuments dont l'histoire et les caractéristiques architecturales sont marquées par les influences françaises et/ou allemandes dues à l'histoire de cette région frontière. Si le patrimoine hérité des périodes anciennes soulève peu de difficultés, il n'en est pas de même pour les programmes liés aux différentes reconstructions de l'Alsace depuis 1871. Aujourd'hui, la question de la valeur patrimoniale et de la représentativité de cette architecture à l'échelle du territoire est au centre des débats. Ainsi, depuis 2008, la CRMH a engagé une étude thématique sur le patrimoine de la reconstruction allemande en Alsace entre 1940 et $1944^{3}$. Il s'agit de mieux connaitre et de valoriser les programmes urbains, industriels ${ }^{4}$ et ruraux engagés durant l'annexion de l'Alsace par le III ${ }^{\mathrm{e}}$ Reich. L'objectif est triple : d'une part, inventorier tous les projets imaginés, d'autre part, en mesurer l'impact en observant leurs réalisations effectives sur le territoire régional ou, du moins, ce qu'il en reste $^{5}$ et enfin, comprendre l'évolution de ces programmes jusqu'à aujourd'hui. L'étude patrimoniale de la Cité paysanne de Marckolsheim en vue de sa protection au titre des monuments historiques a été confiée par la CRMH comme sujet de stage, durant six mois, à trois étudiants. L'objectif de cette équipe pluridisciplinaire était d'élaborer une méthode 
au croisement des compétences d'une architecte, d'une historienne et d'un ethnologue ${ }^{6}$. Ils étaient encadrés par un comité de pilotage réunissant des acteurs du patrimoine régional et transfrontalier, des chercheurs du service de l'Inventaire du patrimoine de la Région Alsace ${ }^{7}$ et de l'université de Strasbourg ${ }^{8}$.

De nombreuses communes sont concernées par des destructions durant les premiers mois de la Seconde Guerre mondiale. Pour autant, toutes n'ont pas fait l'objet d'un programme au sens d'une véritable planification urbaine et architecturale et de la mise en œuvre de moyens pour les réaliser. Celui-ci concerne les Neuordnungsgemeinde (communes de l'ordre nouveau). Selon un document officiel retrouvé dans les archives municipales de Marckolsheim et daté du 3 janvier 1942, il concerne trente-six communes alsaciennes. Elles sont réparties selon un ordre de priorité d'intervention en fonction de leur niveau de destruction, de leur importance agricole et de leur taille. Par ailleurs, elles sont regroupées dans trois Landkreis (arrondissements), dont celui de Sélestat auquel est rattachée Marckolsheim qui compte parmi les dix-sept communes de niveau d'intervention prioritaire. Le bureau de la reconstruction, producteur des documents relatifs à la Cité paysanne est lui, situé à Colmar ${ }^{9}$. Si nous avons choisi d'engager la démarche de protection du patrimoine rural hérité de ce programme par la Cité paysanne de Marckolsheim, c'est d'abord pour la taille et la qualité du projet dans la mesure où ce quartier de quatorze fermes se révèle être le plus conséquent à l'échelle régionale. Ce choix se justifie ensuite par la place de Marckolsheim dans le discours des autorités allemandes de l'époque, présentée comme une reconstruction modèle, un véritable événement à l'échelle du territoire annexé. Enfin, il répond à l'intérêt des habitants et des élus locaux qui nous ont accompagnées dans les recherches et à leur volonté d'appropriation de cette histoire récente.

\section{Protéger la Cité paysanne au titre des monuments historiques ou la nécessaire définition d'une méthode d'inventaire du bâti adapté à un patrimoine spécifique}

Marckolsheim, situé au sud de Strasbourg, et à $14 \mathrm{~km}$ au sud-est de Sélestat, est le cheflieu du canton du même nom. Véritable bourg à partir du milieu du XIX ${ }^{e}$ siècle, c'est une commune catholique. Outre son hôtel de ville datant de 1842, dont les plans ont été établis par l'architecte de l'arrondissement de Sélestat, Antoine Ringeisen, on y trouve une école de fille construite en 1854, une prison et un tribunal datés de la seconde moitié du XIX ${ }^{e}$ siècle. Cette commune rurale se développe à l'intérieur de l'ancien fossé médiéval en eau (Stadtgraben) et compte de nombreuses exploitations agricoles dont nous aurons l'occasion de reparler. Une partie de sa population travaille, depuis la Révolution industrielle, dans les usines des communes avoisinantes et jusqu'à Strasbourg, grâce à une desserte en tram. En 1936, Marckolsheim compte 1810 habitants, population qui ne cesse d'augmenter jusqu'à nos jours. Son tissu bâti est composé de plusieurs ensembles urbains et architecturaux cohérents dont le quartier de la Cité paysanne qui est implanté au nord-ouest du centre ancien et les édifices de la rue principale (rue du MaréchalJoffre) en grande partie reconstruits dans les années 1950 par les architectes français Albert Horn et Auguste Sigrist (fig. $\mathbf{n}^{\circ} \mathbf{1}$ ). 
Figure 1

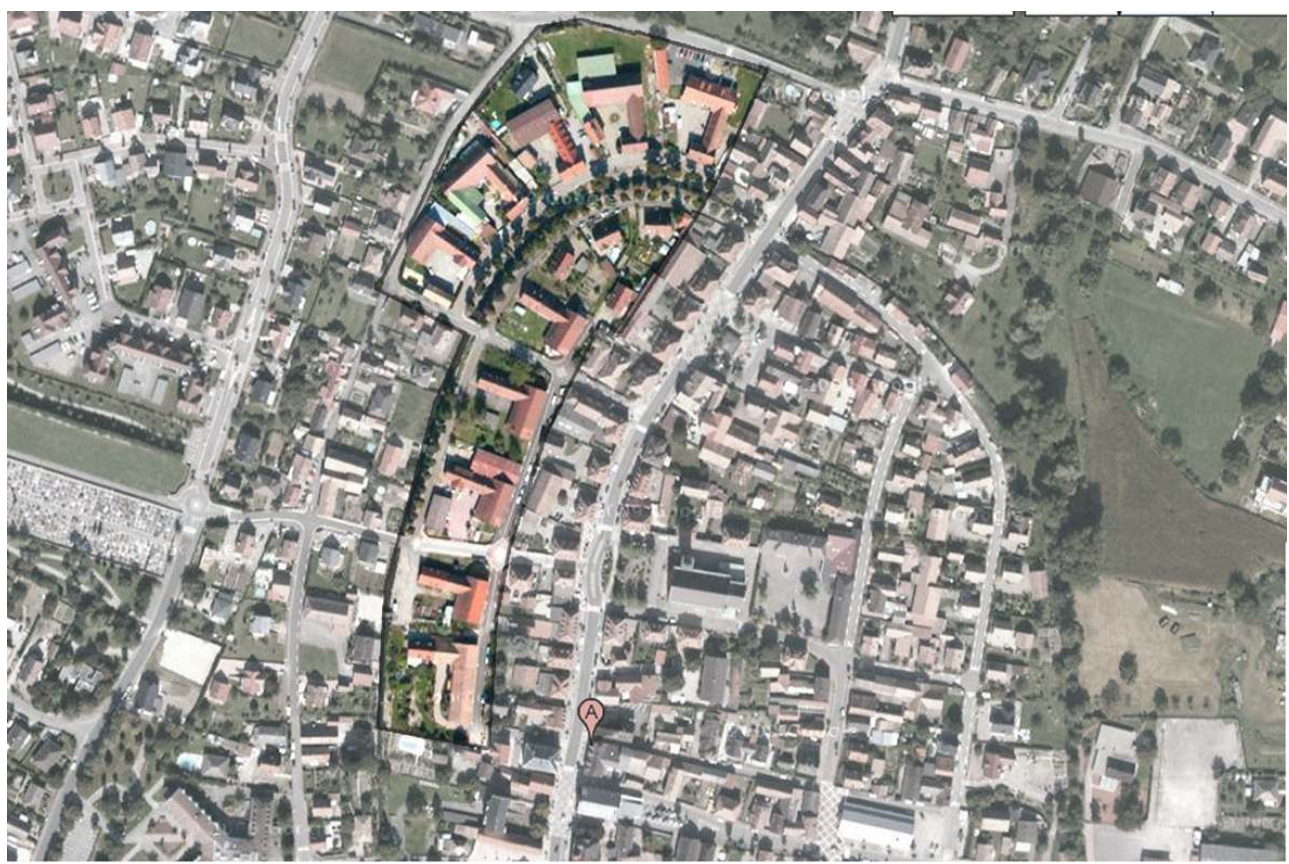

Repérage de la Cité paysanne.

Phot. Aérienne (google maps). Séverine Wodli, 2010. @ DRAC Alsace.

La complémentarité des compétences réunies pour l'étude patrimoniale de la Cité paysanne de Marckolsheim s'est tout d'abord traduite dans l'élaboration d'une méthode adaptée pour en appréhender tous les aspects. Le travail d'analyse tient compte de l'échelle du bourg, du quartier, de l'exploitation agricole puis des bâtiments eux-mêmes. La connaissance fine du bâti est nécessaire à travers la détermination des éléments architecturaux originels et la compréhension de leurs évolutions. C'est pourquoi l'ensemble des archives municipales de cette période a été dépouillé. Celles-ci contiennent notamment, les listes des foyers de Marckolsheim, des supports de propagande, plusieurs plans du bourg portant des informations sur les dégâts subis, les possibilités de reconstruction, les projets de transformation du réseau viaire et les plans d'origine de plusieurs fermes dont celles de la Cité paysanne. Les jeux de "plan-type " conservés qui détaillent les trois modèles de fermes du quartier (Erbhof, Mittlere Landwirt ou Mittlere Betrieb ${ }^{10}$, Arbeiter Wohnhaus) sont signés du bureau de la reconstruction de Colmar. Ils se déclinent en plans de niveaux des bâtiments (logis et dépendances agricoles), en élévation, en coupes transversales et en détails d'exécution. Ils ne comportent pas de signature d'architecte ${ }^{11}$. L'étude doit alors établir des comparaisons entre le projet urbain et architectural tel qu'il apparaît dans les archives et la réalité du terrain.

La phase suivante consiste en la réalisation d'un inventaire du bâti : des relevés, ferme par ferme, permettent de dresser des fiches individuelles liées à une base de données photographiques. Chaque fiche d'identité est complétée par un jeu de plans annotés qui présentent ces spécificités de terrain. Le diagnostic architectural, urbain et technique de la Cité paysanne est ainsi constitué. Il documente l'écart entre le projet de 1940, sa réalisation et son évolution ultérieure jusqu'à aujourd'hui. Tous les éléments constitutifs du programme ont été relevés: matériaux, éléments de modénature des façades, 
équipements du logis, traitement des dépendances agricoles, etc. Enfin, pour confronter et compléter ces informations, des entretiens ${ }^{12}$ ont été organisés avec les habitants ${ }^{13}$ ainsi que quelques personnes ressources ${ }^{14}$. L'aspect sociologique de cette méthode doit nous permettre de comprendre comment l'on travaillait et l'on vivait dans ces fermes, puis de confronter ces renseignements avec le programme et la propagande qui en avait été faits à l'époque.

Au-delà de l'étude de chacune des fermes, l'objectif de cette méthode, est d'appréhender le quartier comme un ensemble bâti cohérent mais dont chacune des parties constituantes n'a pas évolué de la même manière. Des cartes de synthèse portant sur l'occupation des bâtiments, leur état de conservation, leurs valeurs historique et architecturale ont été réalisées afin de proposer une critique d'authenticité et d'émettre des préconisations visant au maintien des qualités patrimoniales de cet ensemble. En effet, la protection au titre des monuments historiques doit permettre de garantir la conservation des éléments architecturaux d'origine identifiés dans un environnement conforme au projet urbain de l'époque, tout en accompagnant l'évolution d'un quartier de bâtiments agricoles dont presque plus aucun ne maintient sa fonction d'origine.

\section{Le contexte : l'annexion de l'Alsace et son intégration au territoire du Reich}

(fig. $n^{\circ} 2, n^{\circ} 3$ )

Figure 2

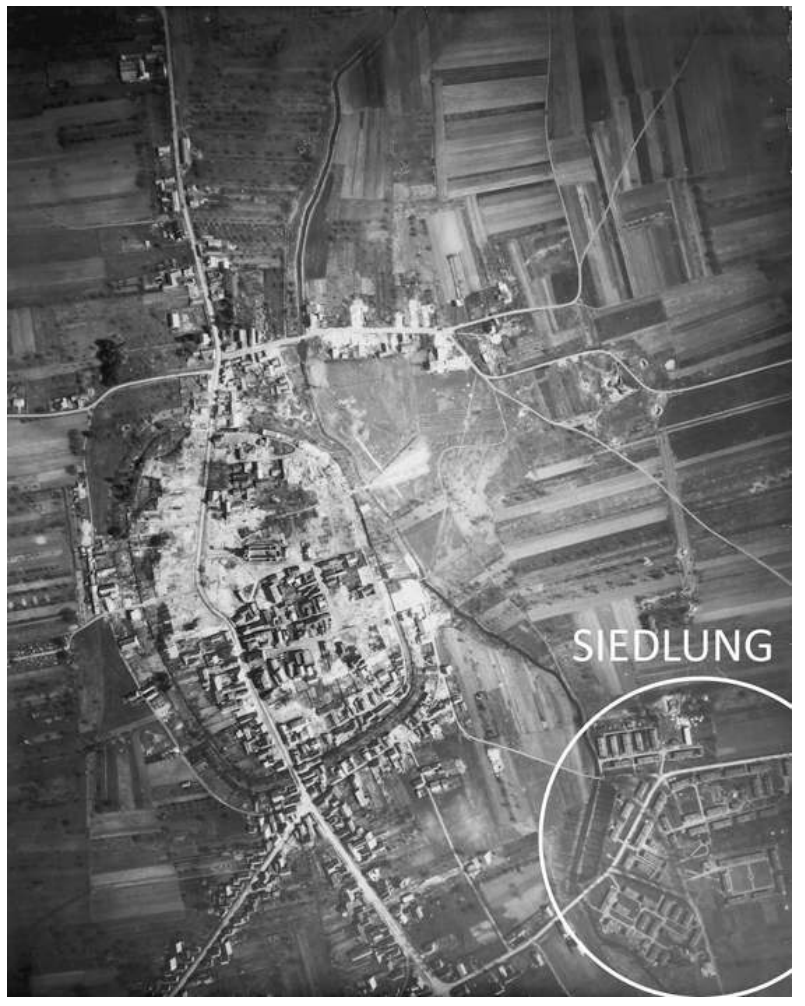

État des destructions de Marckolsheim en 1940 et localisation de la Siedlung.

Phot.aérienne, 1940. ( ) Archives municipales de Marckolsheim (AMM). 
Figure 3
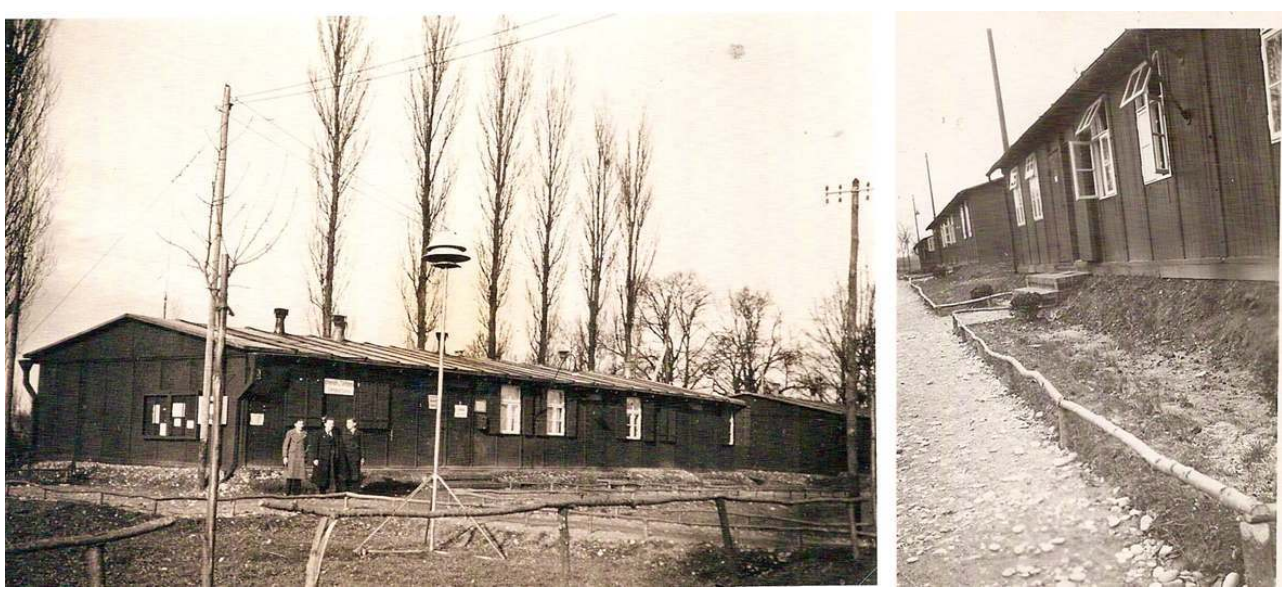

Les baraquements de la Siedlung. Phot 1941

Archive privée. (c) Raymond Baumgarten.

La plus grande partie des Alsaciens se réfugie dans le sud-ouest de la France dès la déclaration de la Seconde Guerre mondiale. À partir du mois de juin 1940, la région devient le théâtre de combats importants. Certains bourgs, comme Marckolsheim, sont rasés à plus de $60 \%$. Dès la signature de l'armistice, le 22 juin 1940, Adolf Hitler ordonne le retour des populations en Alsace et promet que le Reich supportera le poids des dommages subis. Les programmes de reconstruction démarrent, alors que la question du statut administratif de l'Alsace n'est toujours pas réglée ${ }^{15}$.

C'est au mois d'août 1940 que les habitants de Marckolsheim reviennent de la commune de Le Bugue en Dordogne. Ils sont hébergés, pour la majorité d'entre eux, dans la Siedlung, une cité provisoire de baraquements. La presse locale salue cette situation; elle parle de la construction « de belles maisons en bois » et décrit la situation de la manière suivante : "Cela fut une bonne idée de construire les nouveaux logements des habitants [...]. Ces solides constructions en bois massif reposent sur de bonnes fondations. Avec leur peinture rouge sombre, elles se fondent bien dans le paysage d'automne. [...] [Les habitants] sont heureux d'avoir un toit au-dessus de leur tête et de pouvoir travailler. Même les familles nombreuses ont pu trouver de la place pour leur progéniture [...]. Un nouveau Marckolsheim surgit, vous savez tous que les logements actuels ne sont que d'un secours provisoire, jusqu'à ce que des maisons en dur soient reconstruites. ${ }^{16}$ " Ce nouveau quartier implanté au sud-est de la commune est composé de soixante baraquements préfabriqués, dont la réalisation est confiée à des soldats polonais, prisonniers de guerre, cantonnés à Sélestat ${ }^{17}$. Ce quartier abrite avant tout des logements mais également les équipements principaux de l'administration, des cuisines, une boulangerie, une laverie, un coiffeur, des écoles, etc. C'est aussi à la Siedlung que se déroulent la plupart des cérémonies officielles en attendant que la Cité paysanne soit terminée (fig. $\left.\mathbf{n}^{\circ} \mathbf{4}\right)$. 

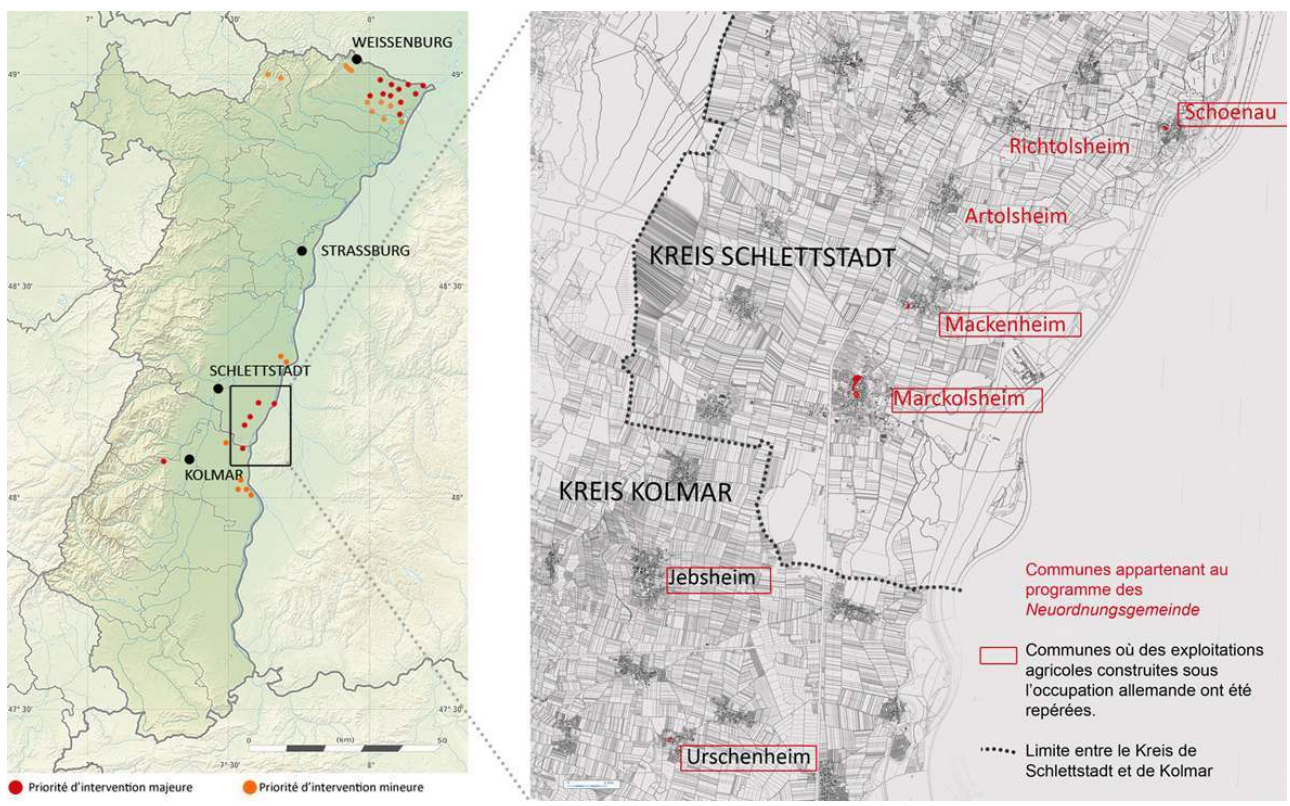

Carte de la répartition des 36 communes de l'ordre nouveau projetées en Alsace d'après la liste publiée en décembre 1941.

Séverine Wodli, 2010. (c) DRAC Alsace.

En parallèle de ces mesures d'urgence, et pour relancer au plus vite un territoire qui doit participer pleinement à l'économie de guerre, l'administration allemande définit le programme des Neuordnungsgemeinde (communes de l'ordre nouveau). Un article du théoricien Friedrich Kann ${ }^{18}$ mentionne à ce sujet la mise en place d'un inventaire des conditions de vie à la campagne en Allemagne au début de l'année 1940, visant à déterminer la situation de chaque famille et à mettre en évidence les problèmes relatifs aux exploitations agricoles. L'objectif est de proposer des solutions pratiques visant à l'amélioration des modes de vies à la campagne comme cela a été ordonné par Richard Walther Darré (1895-1953), ministre de l'agriculture et de l'alimentation du Reich de 1933 à $1942^{19}$. La personnalité chargée de l'application générale de ce programme en Alsace est le Gauleiter Robert Wagner (1895-1946), chef de l'administration civile ${ }^{20}$. À la tête de l'ensemble des services d'architecture, il est à même d'engager les procédures de remembrement qu'il estime nécessaire, de prescrire ou d'interdire la reconstruction de certains bâtiments, de retirer ou de limiter le droit de propriété. Le discours qu'il prononce en juillet 1941 est très explicite: «En mai 1940 les importants combats commencent dans l'ouest, et la plupart des Alsaciens ont pensé qu'ils ne pourraient jamais revoir leurs habitations. Mais la rapidité de la réussite allemande a presque complètement épargné la campagne des destructions de guerre. L'Allemagne n'attend pas la fin de la guerre. L'Allemagne n'attend pas le paiement des dommages de guerre. L'Allemagne travaille ! ${ }^{21}$ »

Sa politique est fixée par l'ordonnance du 2 décembre 1940 intitulée « Mesures pour la réparation des dommages de guerre dans le cadre des communes de l'ordre nouveau ${ }^{22}$ » qui en fixe le cadre. Il s'agit non seulement de prévoir la reconstruction architecturale des villes et villages détruits mais aussi de définir un programme politique et idéologique visant à la "réorganisation fondamentale de la population rurale dans le domaine politique, social et économique, avec un accent particulier mis sur les exigences issues de 
la conquête des espaces étendus à l'est ${ }^{23}$ ». Pratiquement, à l'échelle locale, le programme de la reconstruction des communes de l'ordre nouveau et donc la construction du quartier de la Cité paysanne relève initialement du ressort du Ministère de l'intérieur badois. Mais la mise en œuvre de la planification et des moyens pour l'engager est difficile depuis Karlsruhe. Le $1^{\text {er }}$ mai 1941 une section pour la reconstruction est affectée auprès du directeur de la planification régionale badoise Karl Feldmann.

Le discours justifiant cette politique se fonde sur la dévalorisation de la France pour engager la «re-germanisation » du territoire alsacien. On veut montrer que les Alsaciens n'ont jamais été français mais qu'ils sont véritablement de souche germanique. La propagande ne manque pas d'exploiter les destructions qui avaient marqué le passage des troupes françaises: elles ne sont pas interprétées comme un phénomène lié à la guerre menée contre l'Allemagne mais, comme une mesure vexatoire prise à l'encontre de la population locale. Elle affirme que les Français avaient montré, par leurs mesures de destruction, qu'ils ne songeaient nullement à revenir. « Deux territoires ont toutefois eu à ressentir l'horreur de la guerre : Lauterbourg et Marckolsheim. Là les Français ont essayé de résister. Là s'est fracassée leur force de guerre par leur mauvaise conduite de la guerre et de leur armée. La guerre se poursuit. [...] Un précédent régime, que nous avons appris à connaître en vingt-deux ans de faillibilité aurait construit quelques baraques " provisoires durablement ». Car, vingt ans après la fin de la guerre, tous se souviennent encore des scandales des dommages de guerre. Bien à propos, car la reconstruction, qui a été réalisée en France après 1918, s'est reposée sur les paiements et services de réparations allemands ${ }^{24}$.»

\section{La Cité paysanne comme le témoignage d'une réorganisation sociale et économique du territoire}

Le programme des communes de l'ordre nouveau définit un moteur de développement du monde rural en s'attaquant à son fondement : le monde agricole. Il vise à limiter l'exode des populations engagées depuis la Révolution industrielle et donc à améliorer les conditions de vie à la campagne. Pour les personnalités déjà présentées qui théorisent ce programme, cela doit passer par la modernisation des techniques de production agricole et par la réorganisation de l'ensemble des composantes de la société. La Cité paysanne de Marckolsheim propose une traduction de cette nouvelle vision du monde rural dans un projet urbain et architectural.

Marckolsheim s'inscrit dans le Grand Ried, un territoire peu habité, caractérisé par de petits villages à l'habitat groupé, distants d'environ 3 à $5 \mathrm{~km}$. L'exploitation de cette vaste plaine inondable se caractérise principalement par de une polyculture intensive basée sur une culture traditionnelle (blé, seigle, avoine, pommes de terre, betteraves, et maiis depuis quelques années) et sur une culture pour l'industrie agro-alimentaire (tabac, houblon, betterave à sucre). Celles-ci s'accompagnent également de l'élevage bovin à stabulation permanente. Néanmoins, pendant la Seconde Guerre mondiale, les céréales prédominent, au détriment de l'élevage et de la culture du tabac présents avant et après guerre $^{25}$. En effet, en période de conflit, la production agricole ne se limite pas à assurer l'autosuffisance alimentaire de l'unité familiale mais elle s'intègre aussi dans l'économie agricole du territoire. Il est enfin important de rappeler ici que, malgré la mise en place d'une agriculture spéculative à partir du $\mathrm{XVI}^{\mathrm{e}}$ siècle, l'économie agricole est peu compétitive en Alsace avant 1940. Cette situation s'explique par le morcellement très 
important des terres lié au système d'héritage basé sur le droit germanique ${ }^{26}$ et par la petite taille des exploitations agricoles (moins de 10 ha en moyenne). L'exemple du village agricole de Hollenbach, (Allemagne, Württemberg) ${ }^{27}$ qui peut être comparée à Marckolsheim fait également état de cette situation. Le projet d'application du programme de remembrement tel que nous allons le développer permet de saisir les enjeux de la réorganisation mise en œuvre (fig. $\mathbf{n}^{\circ} 5$ ).

Figure 5
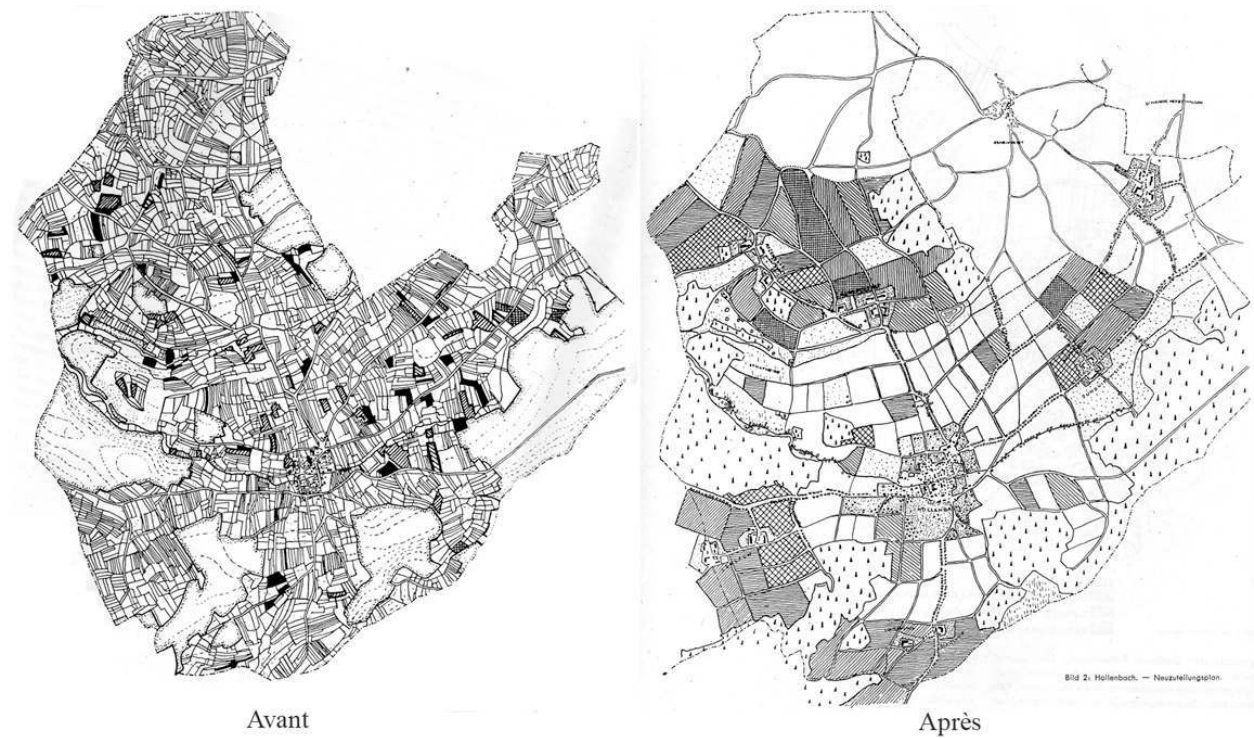

La répartition des terres agricoles de la commune de Hollenbach (Württemberg), avant et après l'application du programme de l'ordre nouveau. Carte extraite de : Neue Dorflandschaften Gedanken und Pläne zum Ländlischen Aufbau in den neuen Ostgebieten und im Altreich. Berlin : Verlag Deutsche Landbuchhandlung Sohnrey \& co, [après 1943], p. 104-105.

Le programme des communes de l'ordre nouveau s'accompagne d'une réorganisation sociale et agraire, qui trouve ses fondements dans la Reicherbhofgesetz (loi sur les exploitations agricoles héréditaires) du 29 septembre 1933, adoptée par le Land de Prusse. Chaque Erbhof (exploitation agricole héréditaire) ${ }^{28}$ doit posséder une étendue minimale de terres pour nourrir une famille, basée sur une unité de mesure appelée Ackernahrung. Ainsi, elle doit en théorie compter entre 5 et 125 ha de terres agricoles. Cette loi fonde par ailleurs la différence de statut entre les exploitants : ainsi elle distingue le Bauer (fermier), celui qui transmet son exploitation de manière héréditaire, du Landwirt (agriculteur). Pour qu'une exploitation soit élevée au rang d'Erbhof, il faut alors remplir plusieurs conditions. Ce privilège est d'abord réservé aux propriétaires allemands par le sang qui ont un attachement idéologique au Reich ainsi que des compétences agricoles reconnues. Ensuite, il faut que soit désigné un héritier unique (cet héritier privilégié est nommé Anerbe) qui n'est pas nécessairement un mâle, ni l'aîné des enfants. Ainsi sera constituée une nouvelle élite paysanne, garante du maintien d'exploitations de grande taille et de la transmission des compétences en matière agricole. Selon les termes de l'historien Wolfgang Voigt, on voit la création d'une «nouvelle aristocratie issue du sang et de la terre ${ }^{29}$ " dont le statut devait pouvoir être remis en cause par le parti nazi en cas de performances « inadéquates $»^{30}$ ou de « déviations sociales et politiques $»^{31}$ (fig. $\left.\mathbf{n}^{\mathbf{6}} \mathbf{6}\right)$. 


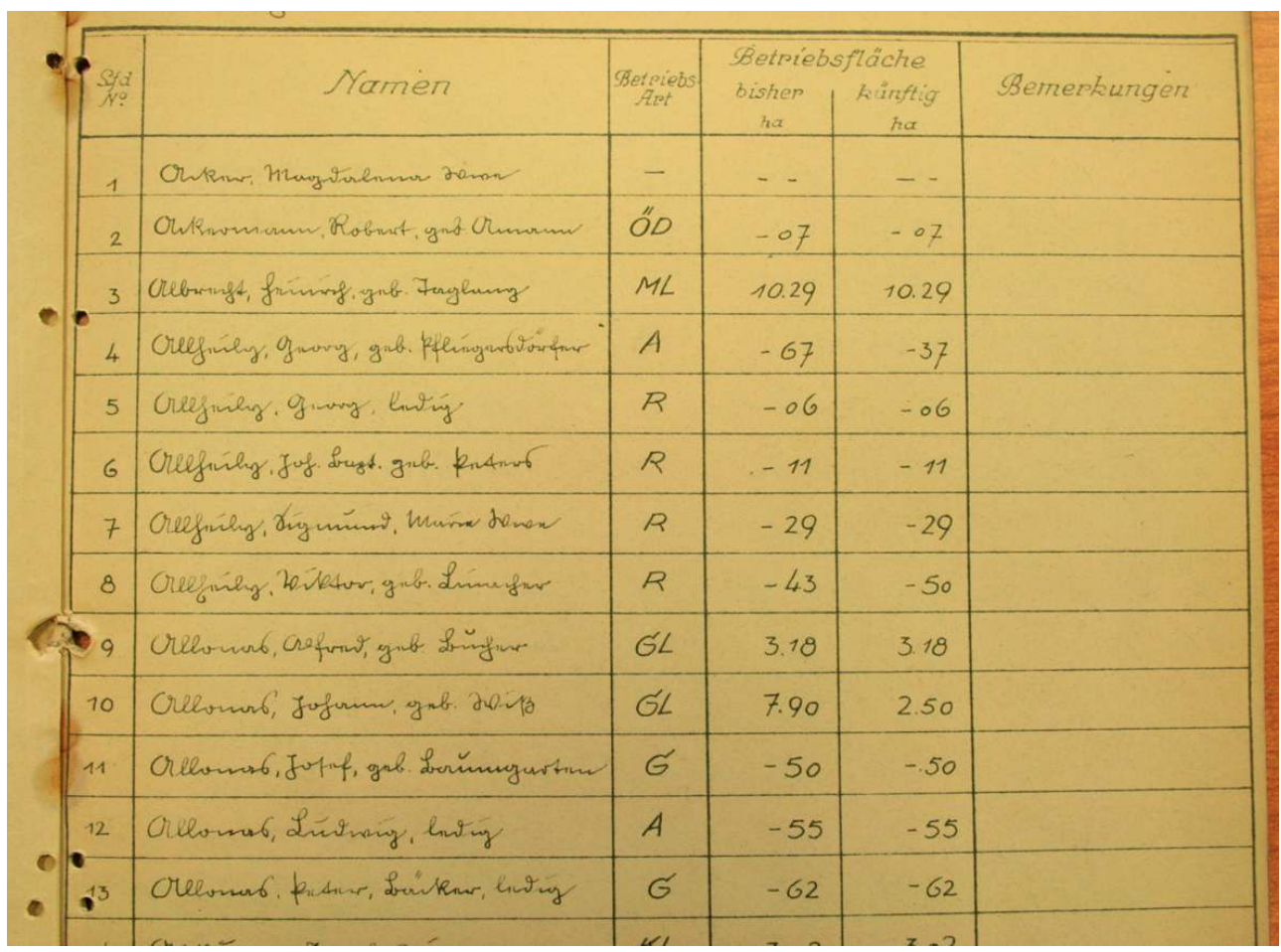

Première page de la liste des foyers de Marckolsheim, présentant la répartition des habitants et mentionnant la composition du foyer, le statut social du chef de famille, le nombre de terres agricoles exploitées et le nombre d'animaux, 1942.

(c) AMM.

La nouvelle organisation de la société agricole par l'application de ce contexte juridique se traduit par la définition de catégories concernant l'ensemble de la société rurale. Pour répondre aux questions que nous nous posions sur la composition de la société de Marckolsheim durant cette période, notamment la hiérarchie des moyens agricoles (fermes et terres cultivées) et leurs modalités d'attribution aux habitants en général et aux paysans en particuliers, nous avons analysé plusieurs listes issues des archives municipales. Nous avons croisé deux listes nominatives datant de 1942 et désignant, entre autres, la qualité de la personne (type d'exploitation, activité professionnelle, etc.), des éléments biographiques (âges des membres de sa famille) et le nombre de terres exploitées. Tous les foyers ${ }^{32}$ sont identifiés. Marckolsheim compte 1232 habitants ${ }^{33}$ pour 373 foyers répartis comme suit :

\section{Caractéristiques de l'ensemble des foyers de Marckolsheim}

\begin{tabular}{|c|c|c|c|c|}
\hline Type d'exploitation & Type d'exploitant & $\begin{array}{l}\text { Nombre } \\
\text { d'exploitants } \\
\text { implantés } \\
\text { dans la Cité } \\
\text { paysanne }\end{array}$ & $\begin{array}{l}\text { Nombre } \\
\text { de chefs } \\
\text { de } \\
\text { foyers }\end{array}$ & $\begin{array}{l}\text { \% rapporté } \\
\text { à } \quad \text { la } \\
\text { population } \\
\text { totale }\end{array}$ \\
\hline
\end{tabular}




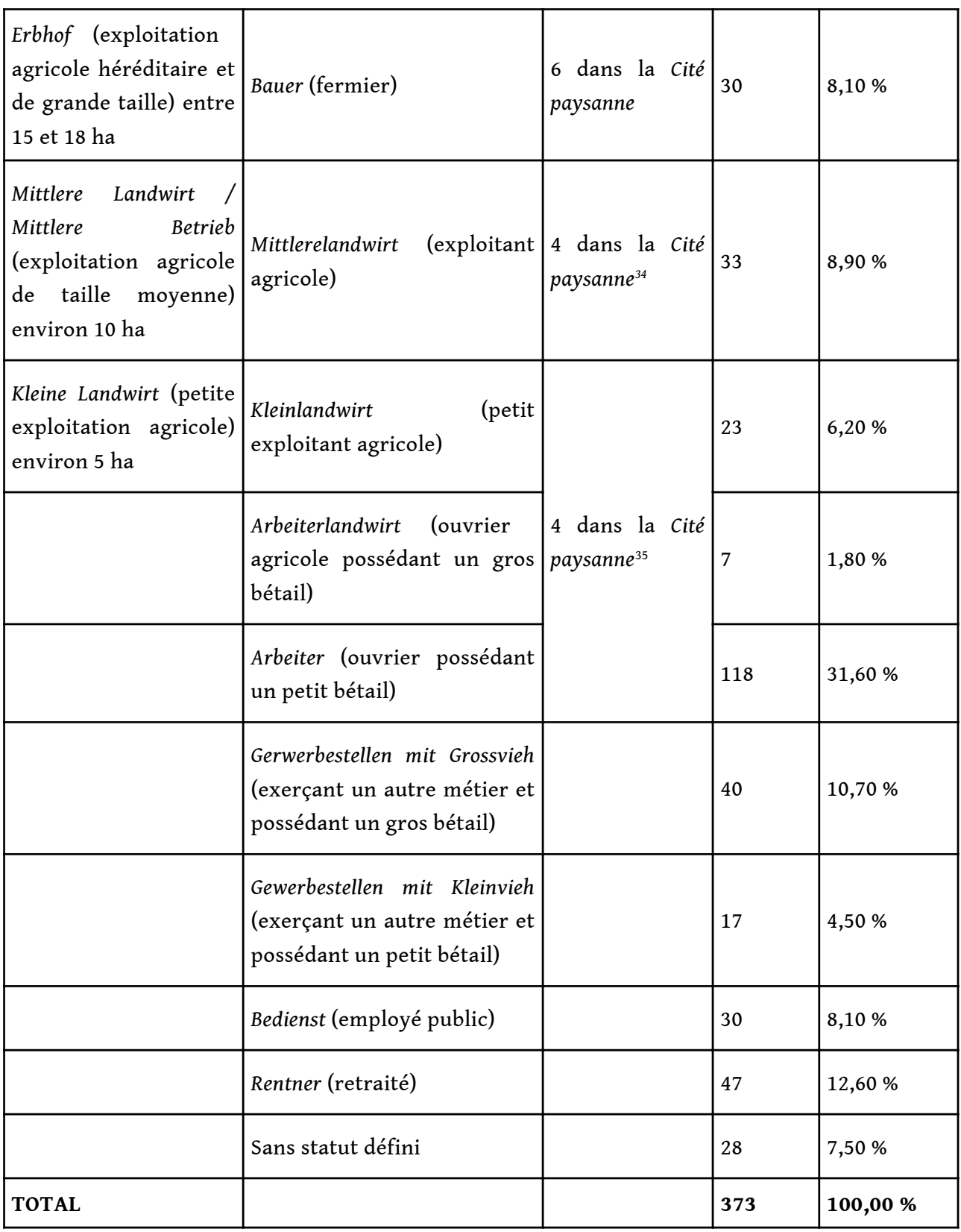

Plusieurs conclusions apparaissent :

- Marckolsheim est une commune dont la population n'est pas composée en majorité d'agriculteurs. En effet 54,9\% des foyers actifs n'exploitent pas de terres agricoles.

- Sur trente Bauern, seuls six résident dans la Cité paysanne. Marckolsheim compte des fermes réparties sur l'ensemble de la commune avant 1939: certaines ont disparu en totalité, d'autres été fortement endommagées mais ont été reconstruites en totalité ou en partie ${ }^{36}$, d'autres encore voient le jour à partir de 1941. Si vingt-quatre Bauer exploitent des Erbhöfe en dehors de la Cité paysanne, cela prouve que toute exploitation agricole répondant aux critères déjà énoncés peut prétendre à ce rang, sans pour autant être une construction neuve. Pour autant, d'après les témoignages recueillis, les exploitations agricoles de la Cité paysanne concentrent les meilleures terres de Marckolsheim et, de fait, concentrent les outils d'exploitations les plus modernes. 
- Six Bauern possèdent une ferme dans la Cité paysanne. Or le quartier ne compte que quatre grandes fermes du type Erbhof et six fermes de taille moyenne du type Mittlere Landwirt. Cela signifie qu'un Bauer pouvait se retrouver à travailler dans une exploitation de taille moyenne qui devenait alors, de fait, une exploitation agricole héréditaire. La taille de la ferme ne constitue ainsi pas un critère suffisant pour déterminer le statut de l'exploitant ${ }^{37}$.

La question de l'attribution des exploitations agricoles, et plus largement de la répartition des terres entre tous les paysans en 1940, a été au cœur de nos interrogations. Cela concernait l'ensemble des fermes, qu'elles soient installées dans la Cité paysanne, ou disséminées sur le ban de Marckolsheim ainsi que la totalité des paysans, quel que soit leur statut. Peu d'informations ont été trouvées dans les archives municipales sur ce sujet et les habitants de la Cité paysanne que nous avons interrogés n'ont pas toujours répondu à nos interrogations. Pour autant, certains critères déterminant les modes d'attribution et de faire-valoir des exploitations sont apparus. En ce qui concerne les terres disponibles, selon les témoignages recueillis, les parcelles agricoles n'ont pas fait l'objet d'un remembrement entre 1939 et 1940. Pourtant, d'après les mêmes sources, le nombre de terres exploitées augmente pendant la guerre. Les archives révèlent en effet que l'administration allemande ${ }^{38}$ décide de défricher certains pâturages au sud du bourg et de déposséder les retraités et employés publics de leurs terres. Ainsi, les paysans ont globalement récupéré leurs terres d'avant-guerre et s'en voient éventuellement attribuer de nouvelles en fonction de leur capacité et de leur statut ${ }^{39}$. En ce qui concerne la hiérarchisation de la société agricole, on peut imaginer qu'une certaine proximité avec les autorités pouvait constituer un avantage. Mais il semble que l'administration allemande tenait également compte des souhaits des exploitants, notamment s'ils étaient élevés au rang de Bauer. Ainsi, un chef de foyer à la tête d'une exploitation agricole héréditaire dont la capacité de travail était réduite pouvait se voir attribuer une exploitation de taille moyenne. C'est pourquoi, comme on l'a vu, certaines Mittlere Landwirt de la Cité paysanne constituent statutairement des Erbhöfe $e^{40}$.

\section{La Cité paysanne, un sujet pour la propagande}

L'exemplarité de Marckolsheim réside également dans le fait que ce village est élevé au rang d'exemple par la propagande du Reich. L'engagement du programme de la Cité paysanne, les différentes manifestations qui l'animent, et la promotion de son plan idéal, sont autant de sujets politiques pour l'administration allemande (fig. $\left.\mathbf{n}^{\circ} \mathbf{7}, \mathbf{n}^{\circ} \mathbf{8}\right)$. 
Figure 7

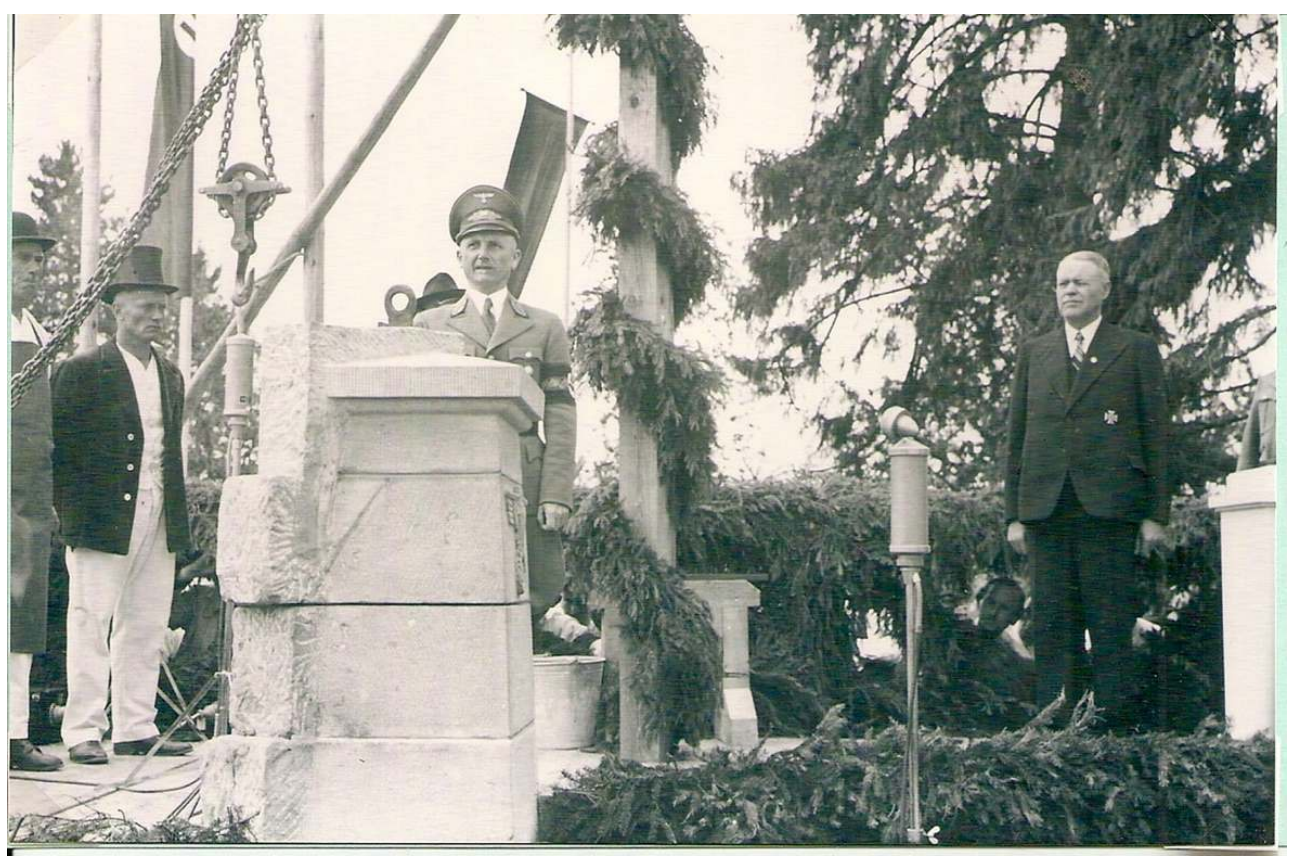

Cérémonie de la pose de la première pierre de la Cité paysanne à Marckolsheim (29 juin 1941). Phot. 1941.

Archive privée. (c) Raymond Baumgarten.

\section{Figure 8}

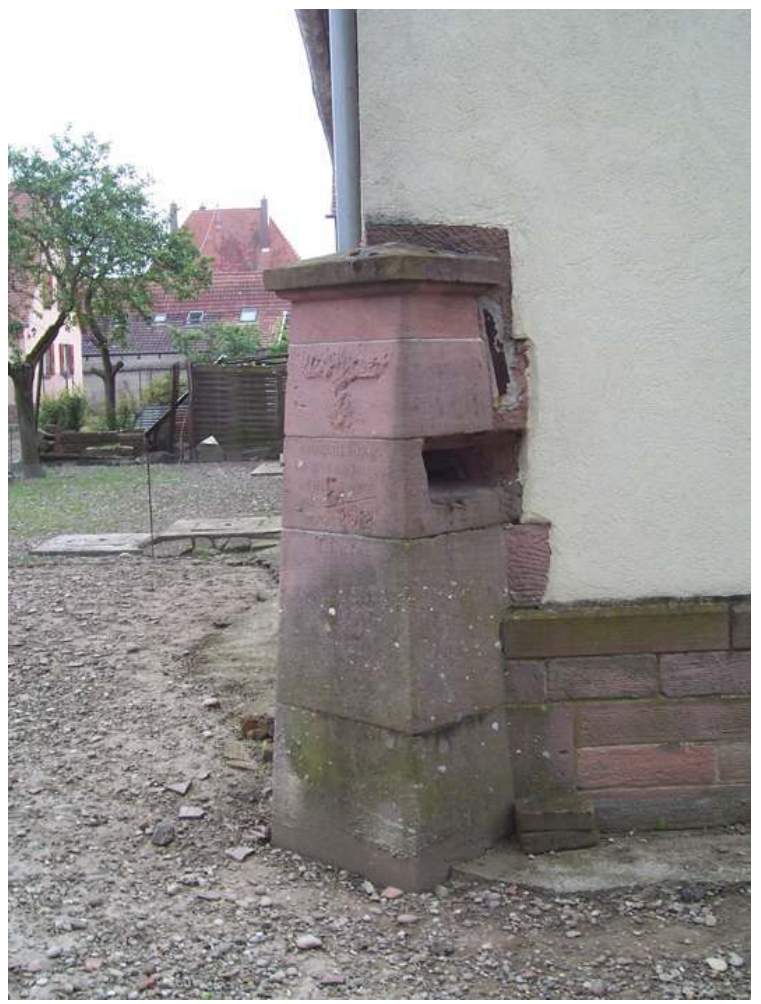

La stèle inaugurale de la construction de la Cité paysanne, située à l'angle nord-ouest du logis de l'exploitation agricole ML6.

PHOT. SÉVERINE WODLI, 2010. @ DRAC ALSACE. 
En effet, voici comment le projet de la Cité paysanne est annoncé dans les Strassburger Neueste Nachrichten du $1^{\mathrm{er}}$ juillet 1941: «Là où la guerre a laissé voir des destructions, après des mois de travail on trouvera à nouveau ponts, rues et maisons. Un mauvais État ne peut réaliser ce genre de chose. Un État, qui reste en guerre, doit donc procéder à de telles reconstructions, lorsqu'il est conscient de réussir. C'est pourquoi la pose de la première pierre à Marckolsheim est un signe de la puissance allemande et d'une réussite consciente allemande. La pose de cette première pierre est aussi l'expression de la venue d'une force, qui ne connaît pas d'obstacles. C'est juste une question de temps jusqu'à ce que la force de la vie allemande ait saisi l'Alsace entière ${ }^{41} »$. Ce journal titre encore : «Pose de la première pierre à Marckolsheim. Le Gauleiter dirige la reconstruction de la commune sinistrée. Une commune modèle est en train de naître ${ }^{42}$ ». Le lancement des travaux de la Cité paysanne est alors un prétexte pour organiser une véritable cérémonie officielle du régime orchestrée par le Gauleiter Robert Wagner. Le samedi 29 juin 1940, toute la population y est invitée. Ces travaux sont célébrés comme le commencement solennel de toute la reconstruction en Alsace. «Samedi sera posée la première pierre à Marckolsheim. Le village détruit sera reconstruit. Et pas seulement! Il doit être plus beau et moderne. Les connaissances nouvelles doivent trouver application dans la reconstruction; les idées nationale-socialistes de l'organisation d'une communauté de village durable sont fondées sur le plan de la reconstruction. Bref, à la place de l'ancien sera érigé un plus beau Marckolsheim. Et bien sûr Marckolsheim n'est pas le seul village où les mains allemandes vont s'employer à reconstruire ${ }^{43}$ ”.

Cette manifestation est parfaitement minutée comme nous l'apprend le programme retrouvé aux archives municipales ${ }^{44}$ : fanfares, chœur du Reicharbeitsdienst (service allemand du travail) et discours sont prévus. Une stèle commémorative en grès rose, visible sur les photographies de l'époque et qui constitue aujourd'hui l'angle nord-ouest de l'une des fermes de la Cité paysanne (celle que nous avons numérotée Mittlere Landwirt 6 ), est inaugurée en grande pompe. À l'intérieur est scellé un document engageant la reconstruction du bourg et signé par certains habitants et représentants des autorités du Reich et de la municipalité ${ }^{45}$. Sa face est sculptée en méplat d'une aigle impériale, aujourd'hui bûchée, et elle est gravée de l'inscription suivante: Grundsteinlegung für der Wiederaufbau im Elsass durch Reichstatthalter Robert Wagner (pose de la première pierre pour la reconstruction en Alsace par le gouverneur Robert Wagner) (fig. $\left.\mathbf{n}^{\circ} \mathbf{9}, \mathbf{n}^{\circ} \mathbf{1 0}\right)$. 
Figure 9

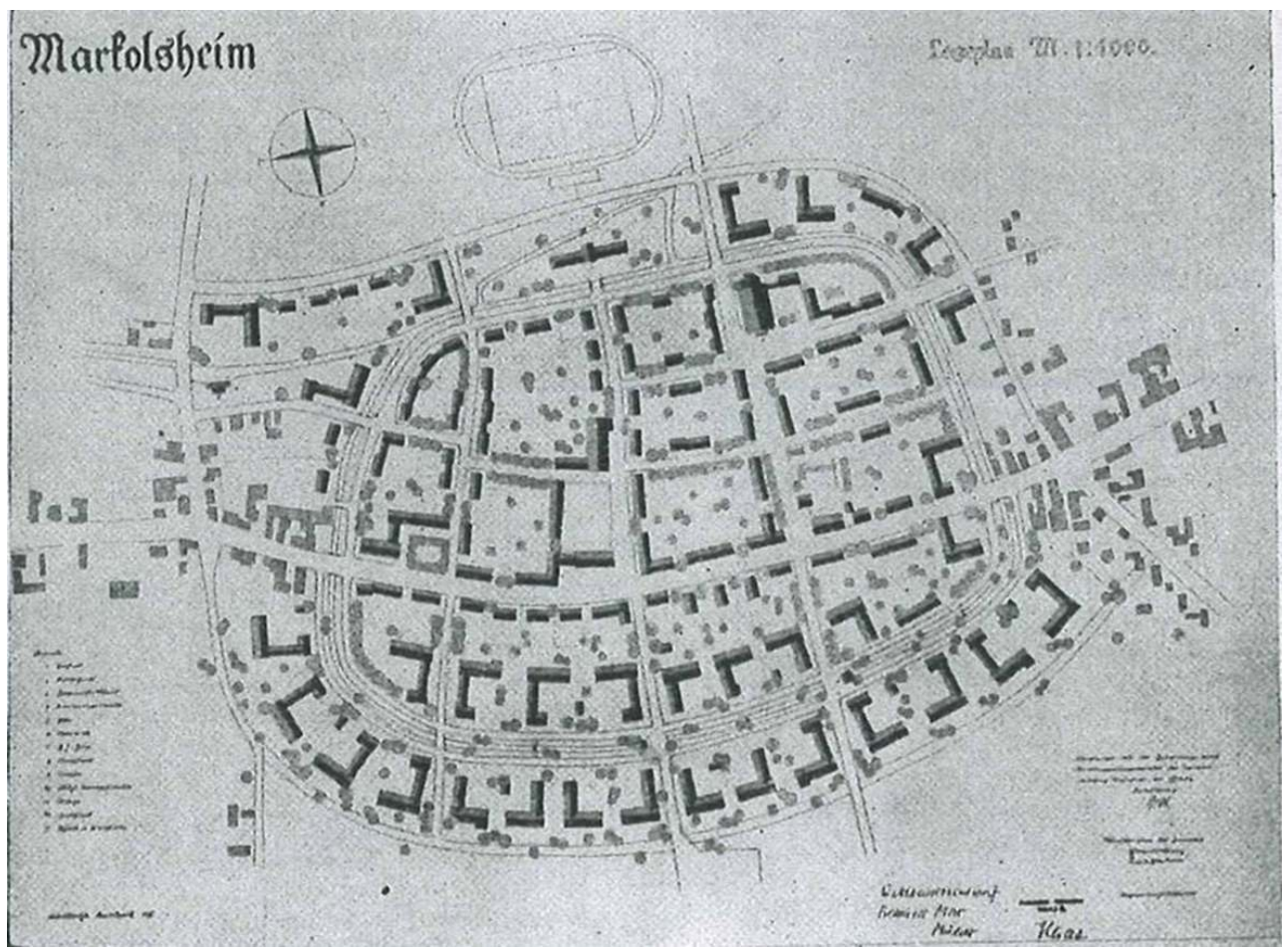

Projet « idéal » de reconstruction du centre-bourg de Marckolsheim. Extrait du Vogesen Heimat Kalender, 1942.

Archive privée. 


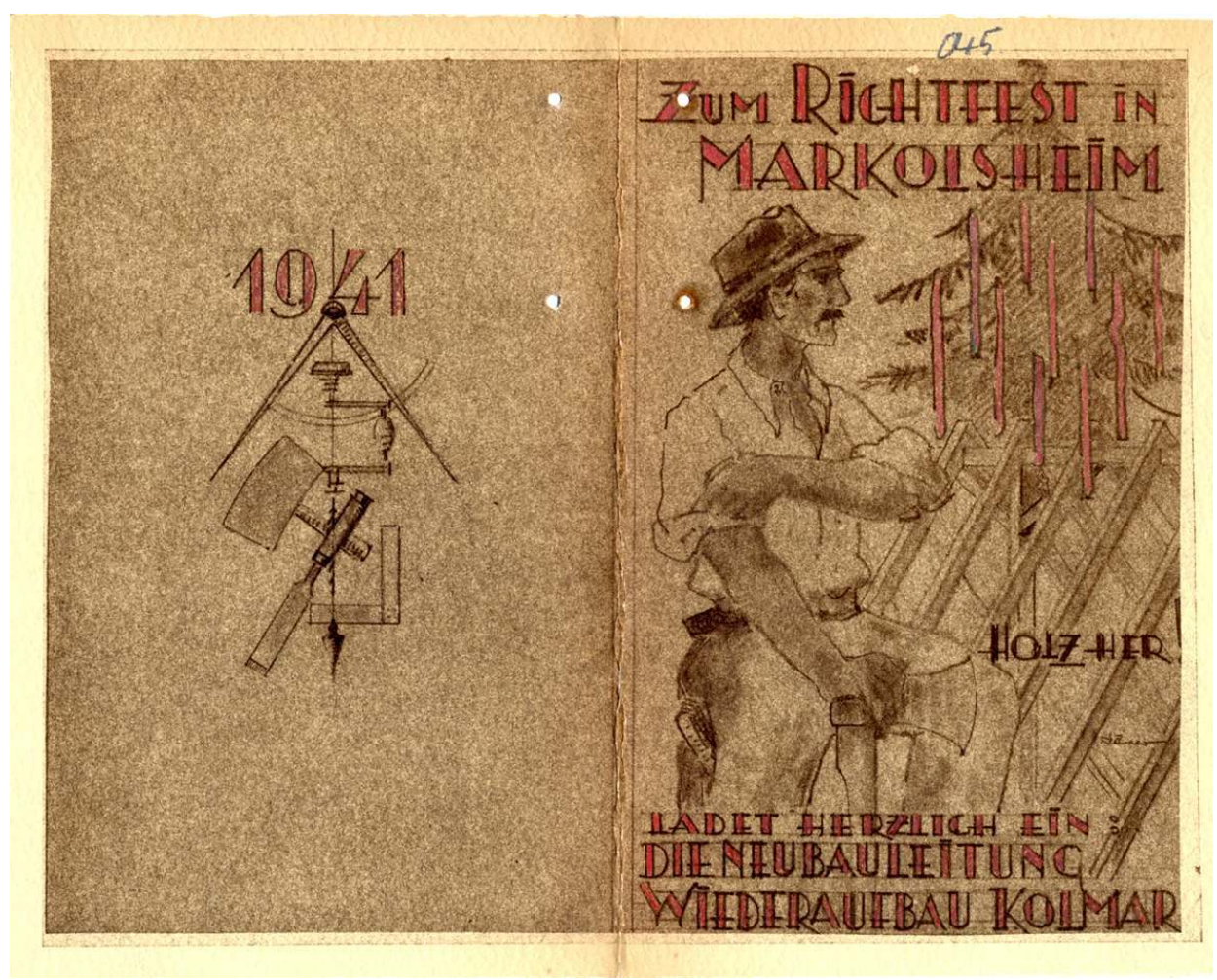

Invitation à la fête des charpentes, dessin, 1941.

(c) AMM.

Hormis, le lancement symbolique du programme de la Cité paysanne, les fêtes qui se déroulent à Marckolsheim sont, comme ailleurs, célébrées dans la presse locale. Elles fournissent l'occasion de réaffirmer des valeurs traditionnelles : la place des agriculteurs ${ }^{46}$ ou la valeur du travail et de l'artisanat ${ }^{47}$. Enfin, la diffusion du plan idéal de Marckolsheim forme un dernier moyen d'utiliser ce sujet particulier pour la propagande. Des plans d'ensemble et de détails sont publiés dans le Vogesen Heimat Kalender ${ }^{48}$ en 1942. Ces documents sont réalisés après de début de la construction des premières fermes qui eut lieu en juin 1941. Pourtant ils ne correspondent pas du tout aux implantations réelles des bâtiments. La partie ouest du centre-bourg est occupée par une douzaine de bâtiments semblant être des fermes de grande taille de type Erbhöfe et une dizaine de fermes de taille moyenne de type Mittlere Landwirt. Finalement, la communication de ce programme est représentative des ambitions de ce que le Reich souhaite pour les communes de l'ordre nouveau : le plus important réside dans l'affirmation des ambitions de modernité, d'hygiène et de confort. Ainsi, ce qui compte, c'est de promouvoir un tissu bâti aéré, une rationalisation des réseaux viaires, une meilleure disposition des fermes en périphérie du bourg et à proximité des terres à cultiver, un regroupement des services et des administrations au centre du bourg dans des bâtiments dont les plans suggèrent une architecture monumentale, etc. 


\section{Le projet de la Cité paysanne}

Le quartier de la Cité paysanne s'inscrit dans un contexte bâti spécifique, qu'il s'agit de comprendre afin de mesurer les enjeux architecturaux et urbains de ce projet et d'en évaluer l'impact dans le tissu bâti ancien. L'architecture rurale traditionnelle du Ried alsacien ${ }^{49}$ que l'on rencontre à Marckolsheim avant 1939 est caractéristique (fig. $\mathbf{n}^{\circ} \mathbf{1 1}, \mathbf{n}^{\circ}$ 12).

Figure 11

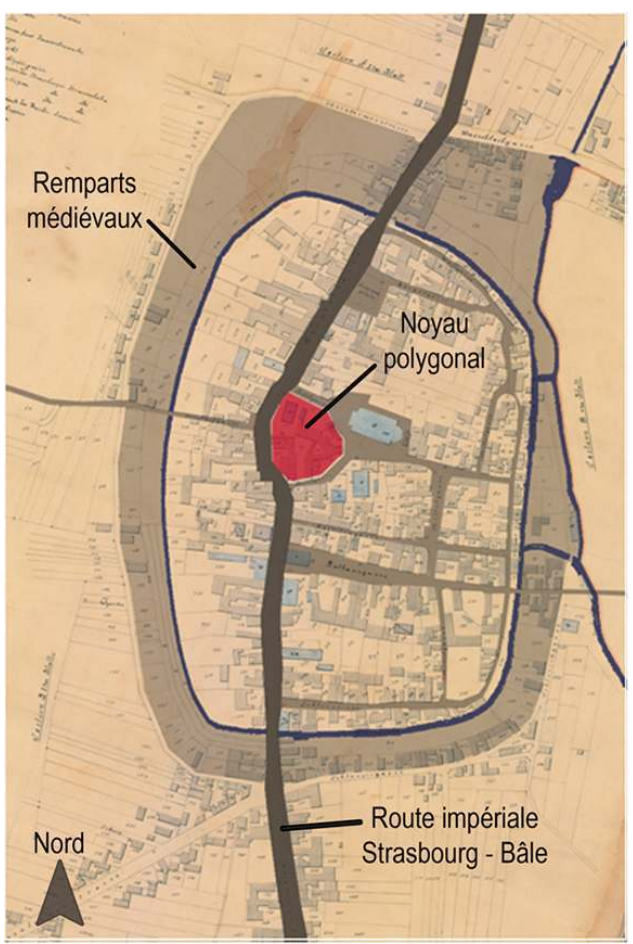

Avant 1940

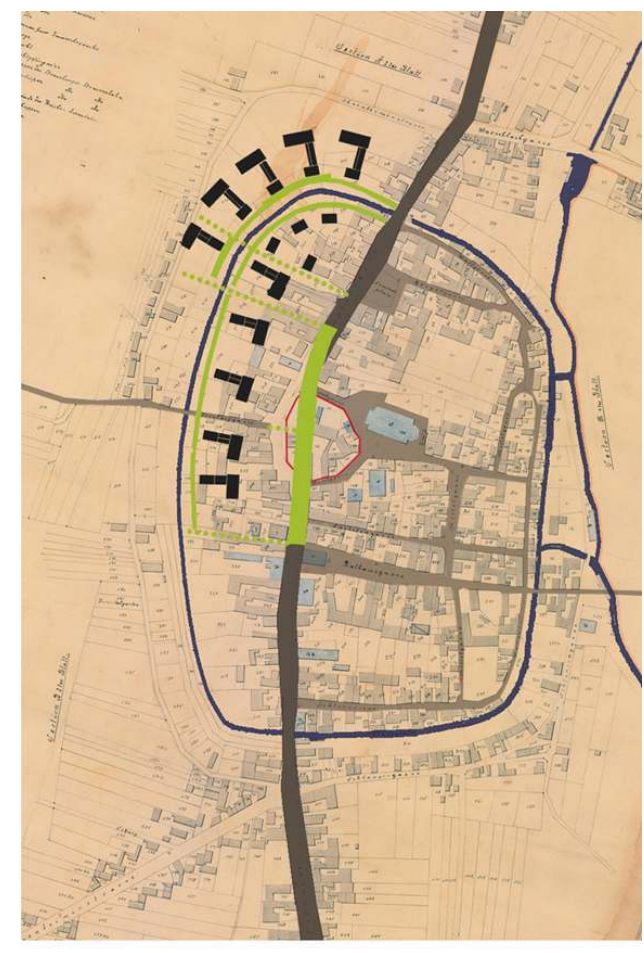

Après 1940

Les modifications urbaines du centre-bourg sous l'occupation allemande. SÉVERINE WODLI, 2010. @ DRAC ALSACE. 


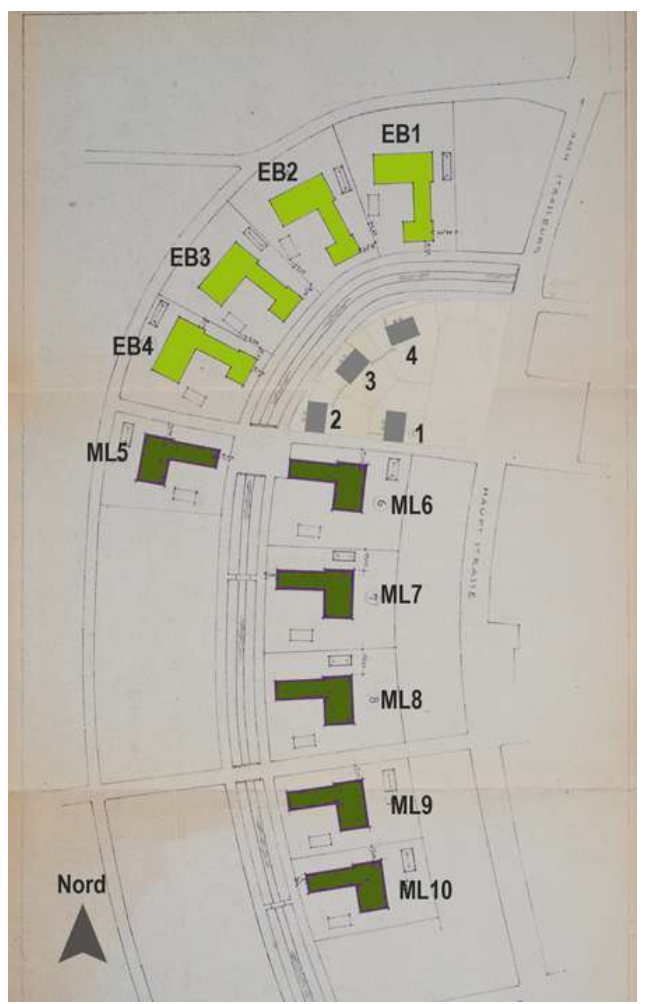

Plan d'aménagement de la Cité paysanne réalisé par le Bureau de la reconstruction de Colmar en juillet 1941 et repérage des fermes.

Séverine Wodli, 2010. (c) DRAC Alsace.

Les représentations cartographiques anciennes de Marckolsheim mettent en évidence trois éléments urbains structurants: le Stadtgraben (fossé en eau des anciens remparts médiévaux), l'ancienne route impériale reliant Strasbourg à Bâle traversant le centrebourg selon l'axe nord-sud et le noyau polygonal constituant l'enveloppe d'un château disparu. Jusqu'à la veille de la Seconde Guerre mondiale, le bâti s'est développé dans l'aire délimitée par la ceinture médiévale sous la forme d'un "village-tas», avec une dissociation du bâti et des terres cultivées. Les exploitations agricoles font partie intégrante du tissu bâti ancien du village. Cette disposition rend difficile l'accès aux terres agricoles, qui nécessite obligatoirement de franchir le fossé en eau.

Sur la parcelle elle-même, les bâtiments de l'exploitation traditionnelle sont distribués autour d'une cour ouverte, dissociée de l'espace public par une clôture légère. Plusieurs dépendances agricoles déterminées par leurs fonctions, contiguës entre elles ou avec le logis (la grande proximité entre les espaces d'habitation et les étables est souvent une généralité) mais également individualisées s'organisent en forme de « $\mathrm{L}$ ». La fosse à purin, creusée au centre de cette cour et la circulation, s'organise depuis les dépendances vers la rue. Le logis est en général implanté en limite parcellaire, perpendiculairement à la rue, offrant l'entrée principale sur la cour. Sans eau courante et sans électricité évidemment, le confort et l'hygiène sont rudimentaires. À cette date, les techniques de productions agricoles sont en Alsace encore très archaïques: l'agriculture demeure traditionnelle et peu mécanisée. Pour ce qui est des matériaux de construction mis en œuvre, le bois reste le plus employé, avec dans certains cas, un soubassement en grès. La 
toiture à deux pans avec une forte pente est recouverte de tuiles plates écailles traditionnelles appelées Bieberschwanz.

Cette situation explique que les choix de composition et de réalisation de la Cité paysanne répondent à la prise en compte de nouveaux critères de planification urbaine et de modernités architecturales. Ce programme permettant la construction ex-nihilo d'un quartier de quatorze fermes, vise à améliorer les qualités de vie et de travail des exploitants agricoles. Ceci passe par la rationalisation des espaces afin de limiter l'effort humain et de garantir la rentabilité économique de l'exploitation. Cette ambition se traduit à toutes les échelles, de l'implantation des constructions au plan même des parties constituantes des fermes. Par exemple, le tracé du réseau viaire est rectifié et le nouveau découpage parcellaire est aéré pour limiter les risques de propagation de maladie et de feu. L'activité agricole détermine la vie du quartier et donc son organisation spatiale : la cour de la ferme s'ouvre sur l'espace public en offrant une vision propre de l'exploitation depuis la rue et à l'arrière sont relégués les accès aux dépendances et le fumier. De plus, les circulations se spécialisent grâce au dessin traversant des parcelles. L'influence du courant hygiéniste se traduit encore par l'installation d'une distance minimale entre la porcherie et le logis, distance variable en fonction du nombre de bêtes. Enfin, si le style régionaliste s'impose, l'introduction de nouveaux matériaux comme le béton, répond aux influences de l'architecture fonctionnaliste.

\section{Aspects urbains et paysagers}

(fig. $\left.n^{\circ} 13, n^{\circ} 14\right)$

Figure 13

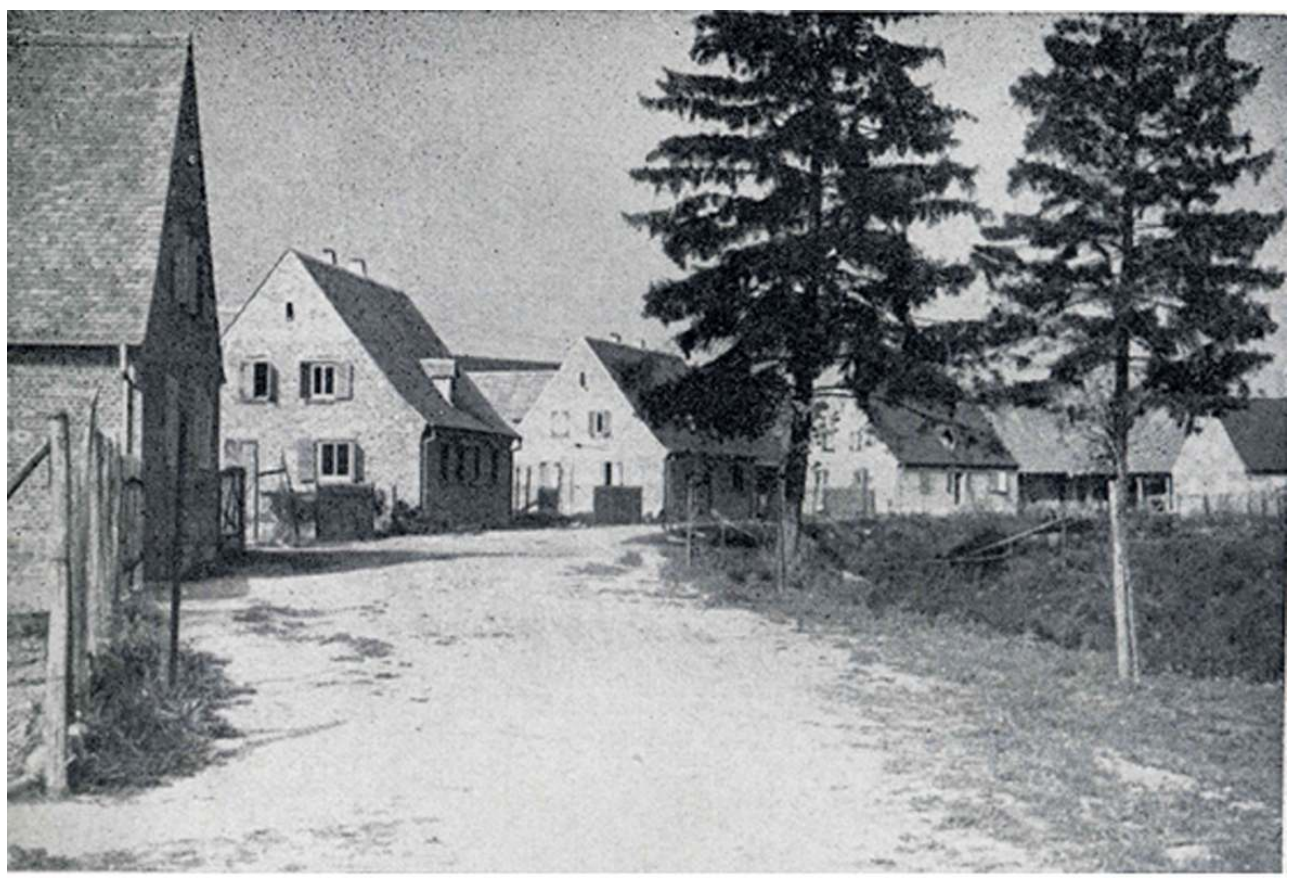

La Cité paysanne en 1945. Phot. extraite de : BOPP, Marie Joseph. L'Alsace sous l'occupation allemande 1940-1945. Le Puy: X. Mappus Éditeur, p. 78. 


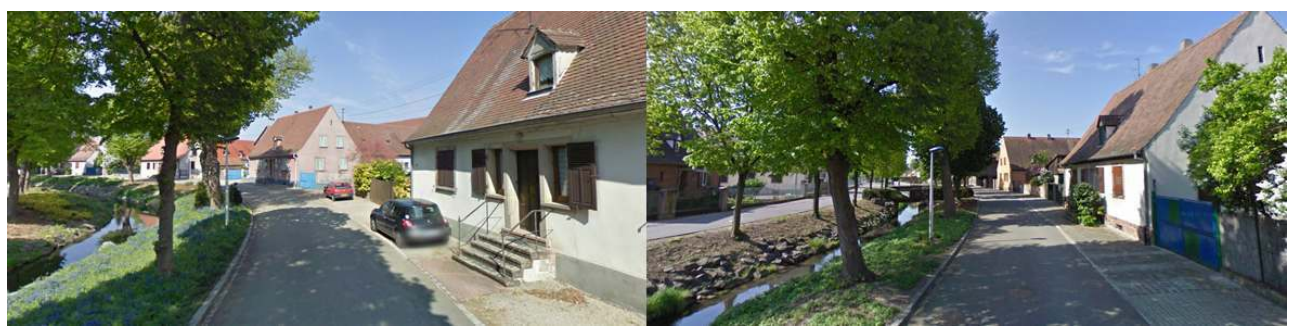

Vue d'ensemble de la Cité paysanne.

PHOT. SÉVERINE WOdLI, 2010. (C) DRAC ALSACE.

\section{Adresses des fermes de la Cité paysanne par modèle :}

\begin{tabular}{|c|c|c|}
\hline & $\mathbf{N}_{50}^{\circ}$ (sur le plan) & Adresses \\
\hline 4 fermes de grande taille (Erbhof) - EB & $\begin{array}{l}\text { EB1 } \\
\text { EB2 } \\
\text { EB3 } \\
\text { EB4 }\end{array}$ & 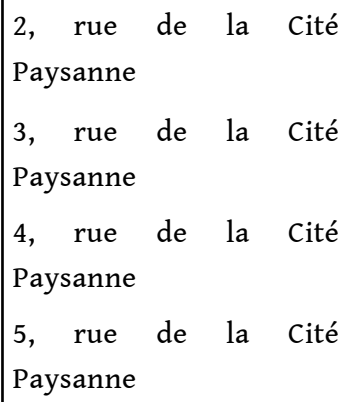 \\
\hline $\begin{array}{l}6 \text { fermes de taille moyenne (Mittlere Landwirt) - } \\
\text { ML }\end{array}$ & $\begin{array}{l}\text { ML5 } \\
\text { ML6 } \\
\text { ML7 } \\
\text { ML8 } \\
\text { ML9 } \\
\text { ML10 }\end{array}$ & $\begin{array}{l}\text { 6, rue de la Cité } \\
\text { Paysanne } \\
\text { 4, rue de Verdun } \\
\text { 9, rue Le Bugue } \\
\text { 17, rue Le Bugue } \\
\text { 19, rue Le Bugue } \\
\text { 21, rue Le Bugue }\end{array}$ \\
\hline 4 maisons d'ouvriers agricoles - AOLZ & $\begin{array}{l}\text { AOLZ1 } \\
\text { AOLZ2 } \\
\text { AOLZ3 } \\
\text { AOLZ4 }\end{array}$ & $\begin{array}{l}14, \text { rue de la Dordogne } \\
5 \text { rue Le Bugue } \\
3 \text { rue Le Bugue } \\
1 \text { rue Le Bugue }\end{array}$ \\
\hline
\end{tabular}

Après les destructions aériennes de 1940, l'administration allemande décide d'implanter la construction de nouvelles fermes au nord-ouest du bourg ancien ${ }^{51}$ de part et d'autre du Stadtgraben du fait des facilités d'aménagement, du potentiel d'extension et également pour jouxter les terres les plus fertiles du ban communal. Les terrains nouvellement lotis correspondent d'après le cadastre de 1909 à d'anciens jardins et au domaine d'un notaire de Marckolsheim. Sur ce plan apparaissent encore quelques vieilles maisons, certainement endommagées, qui seront également détruites. Il est dorénavant possible d'intervenir de manière radicale sur le plan d'ensemble: l'ancien noyau polygonal disparaît, le tracé de l'ancienne route impériale est rectifié pour être rectiligne, des fonds de parcelle sont bâtis, des parcelles dédiées aux vergers et aux jardins sont urbanisées, 
des nouvelles rues sont créées Ainsi, l'accès à la ferme se fait par une voie principale, tandis que la grange possède deux entrées l'une donnant dans la cour et une seconde sur une voie de desserte à l'arrière (actuelles rue de la Cité-Paysanne et rue Le-Bugue). Ce dispositif facilite le travail agricole et détourne le trafic agricole de l'axe principal. Par ailleurs, l'actuelle rue de Verdun n'a pas été construite à son emplacement projeté. Elle se situe entre la ML6 et la ML7 au lieu de marquer la séparation entre les trois types de constructions. Une grande place, franchissant le fossé, avait été également été envisagée au droit de la stèle commémorative, probablement pour mettre en valeur celle-ci, mais le projet n'a pas abouti, peut-être par faute de temps (fig. $\mathbf{n}^{\circ} \mathbf{1 5}$ ).

Figure 15

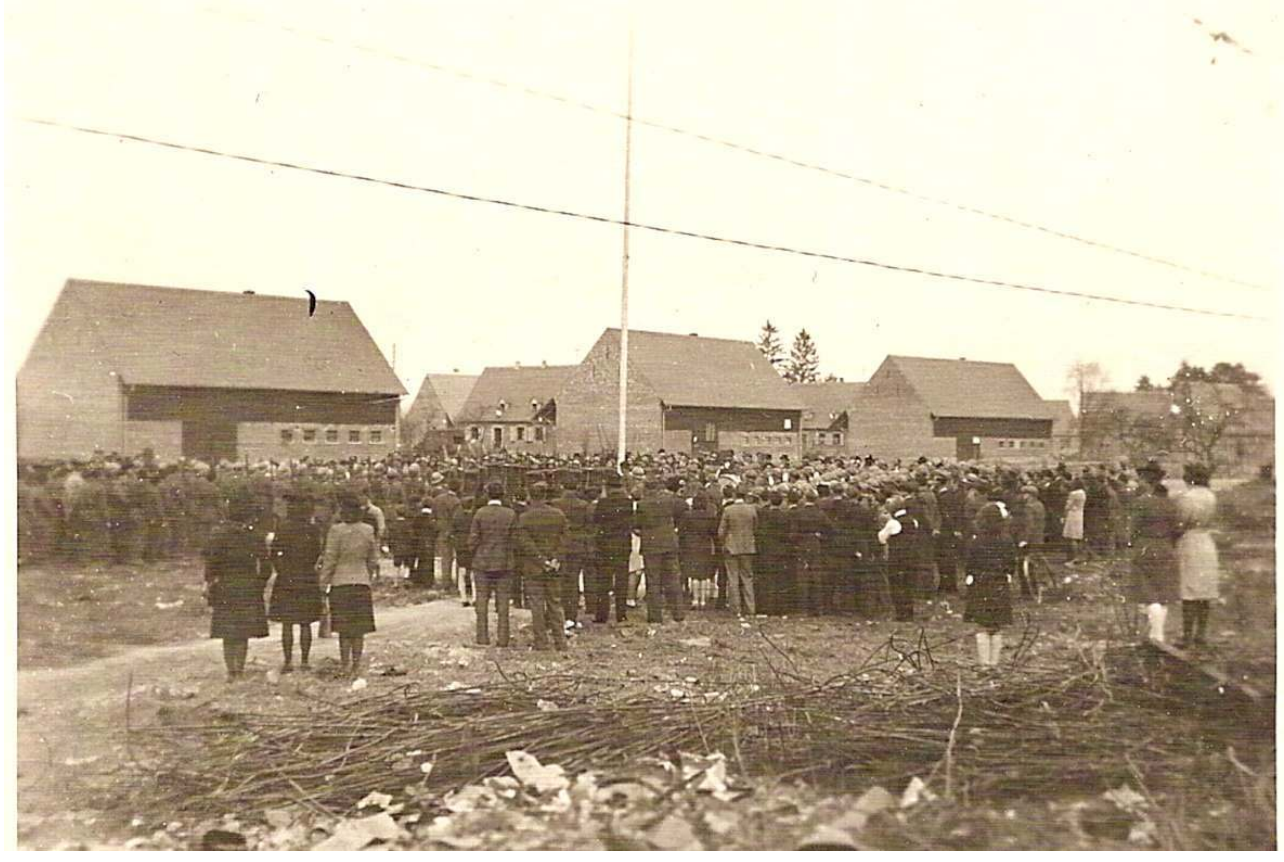

Vue d'ensemble des granges par l'arrière au moment de l'installation des premiers habitants dans la Cité paysanne. Phot 1942.

Archive privée. (c) Raymond Baumgarten.

On compte moins de deux années (de juin 1941 à avril 194352) entre la date de réalisation des plans d'aménagement urbain et l'installation effective des premiers habitants : un temps finalement assez court au regard du contexte économique et de l'ampleur des travaux. Le plan d'aménagement urbain du juillet 1941 montre que la Cité paysanne a été construite en deux tranches de travaux : la première comprend les quatre grandes fermes de type Erbhof ainsi que les six fermes moyennes de type Mittlere Landwirt, et la seconde consiste en la construction des quatre Arbeiter Wohnhaüser ${ }^{53}$. L'ordre de construction des bâtiments a pu être partiellement déterminé grâce aux entretiens avec les habitants et aux photographies d'époque. Les nouvelles parcelles sont grandes, elles offrent des surfaces équivalentes à 20 ares et un coefficient d'occupation du sol inférieur à 0,5 , ce qui contraste avec la densité du centre ancien et les fermes d'avant 1939. De plus, l'implantation du logis avec un recul de $5 \mathrm{~m}$ par rapport à la rue rompt avec les traditions constructives où, comme on l'a vu, le logis était implanté en limite parcellaire sur rue (f ig. $\left.n^{\circ} 16, n^{\circ} 17\right)$. 
Figure 16
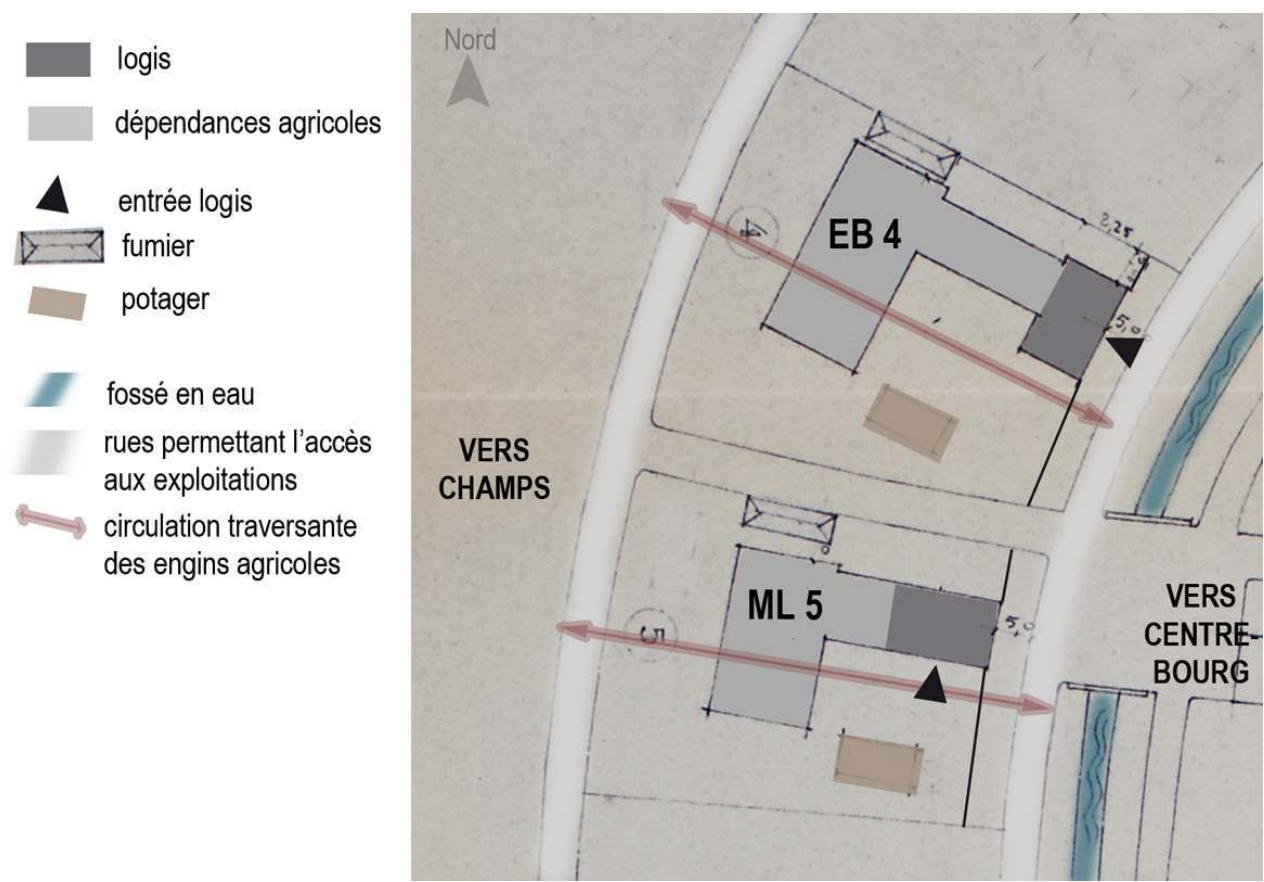

Comparaison de l'organisation de la parcelle pour une Erbhof et une Mittlere Landwirt.

SÉVERINE WODLI, 2010. (C) DRAC ALSACE.

Figure 17

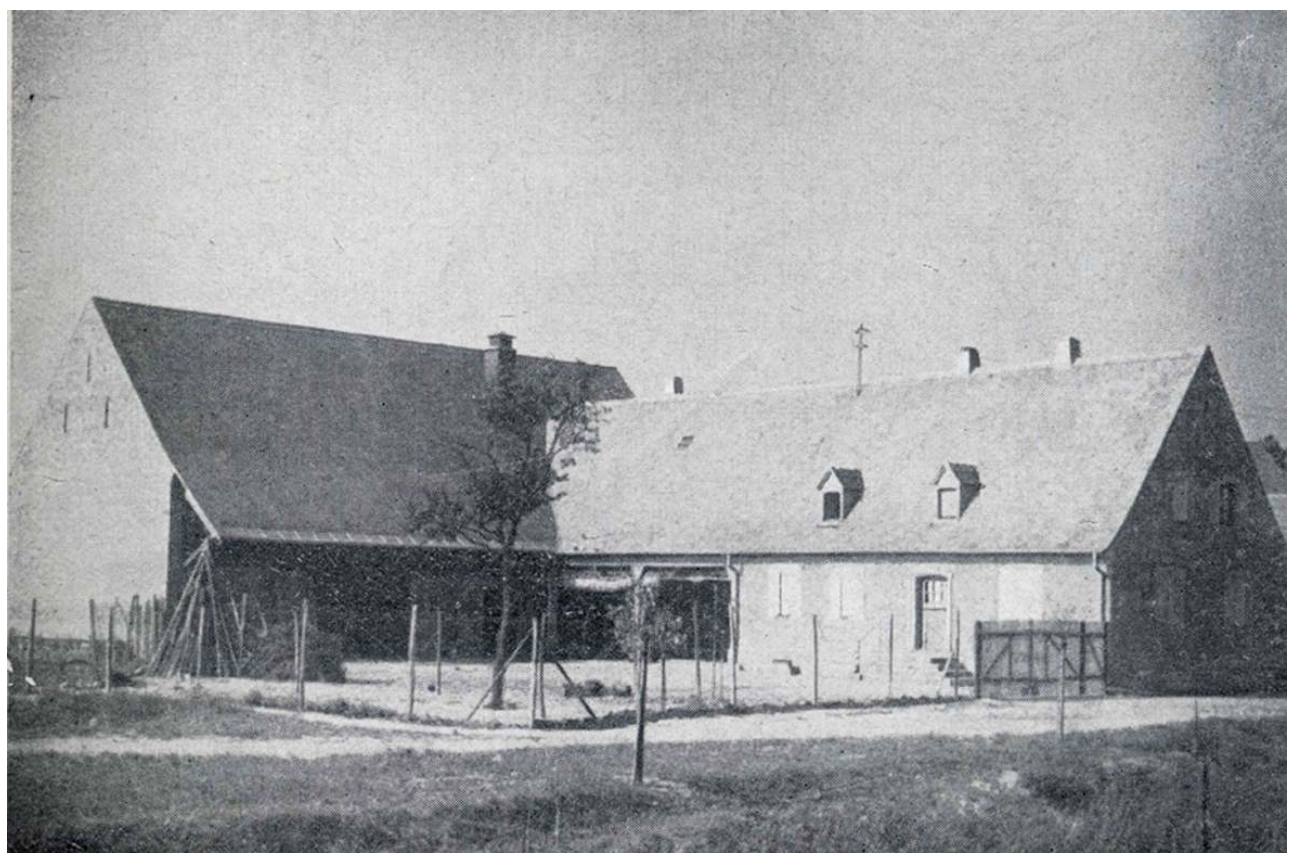

Une exploitation agricole de type Mittlere Landwirt (ML5) à la fin de la Seconde Guerre mondiale. Phot. extraite de : BOPP, Marie Joseph. L'Alsace sous l'occupation allemande 1940-7945. Le Puy : X. Mappus Éditeur, p78.

Les nouvelles parcelles sont grandes. Elles offrent des surfaces équivalentes à 20 ares et un coefficient d'occupation du sol inférieur à 0,5 , ce qui contraste avec la densité du 
centre ancien et les fermes d'avant 1939. De plus, l'implantation du logis avec un recul de $5 \mathrm{~m}$ par rapport à la rue rompt avec les traditions constructives où, comme on l'a vu, le logis était implanté en limite parcellaire sur rue. Une clôture légère, composé de lattes verticales de bois, est réalisée dans le prolongement du logis, sans que celle-ci ne correspondent à la limite parcellaire entre la rue et l'espace privé. La distribution sur la parcelle d'une Erbhof et d'une Mittlere Landwirt varie peu. Ces exploitations sont composées de trois entités bâties accolées s'articulant sous la forme d'un « L » : le logis, la porcherie et la grange. Les volumes des dépendances agricoles ont été dimensionnés en fonction de la superficie de terres agricoles cultivées et du nombre de têtes de bétail attribué à chaque exploitation. La cour est une sorte de " place publique domestique $"^{54}$ par opposition à l'arrière de la grange exclusivement destinée à la circulation des machines agricoles, à la fosse à purin et au potager orienté plein sud. La cour en terre battue ne présente à l'origine aucun traitement du sol spécifique. Le plan des ML est fidèle au "plan-type » du bureau de la reconstruction. Seul l'accès au surcroît de la porcherie de la ML7 diffère : son escalier est implanté parallèlement au mur du logis alors que dans les autres cas, il est perpendiculaire au mur conformément au plan d'exécution. Enfin, on observe une continuité des circulations, aussi bien en intérieur qu'en extérieur, le long des dépendances agricoles côté cour, avec une circulation couverte par un grand débord de toit (fig. $\left.\mathbf{n}^{\circ} \mathbf{1 8}\right)$.

Figure 18

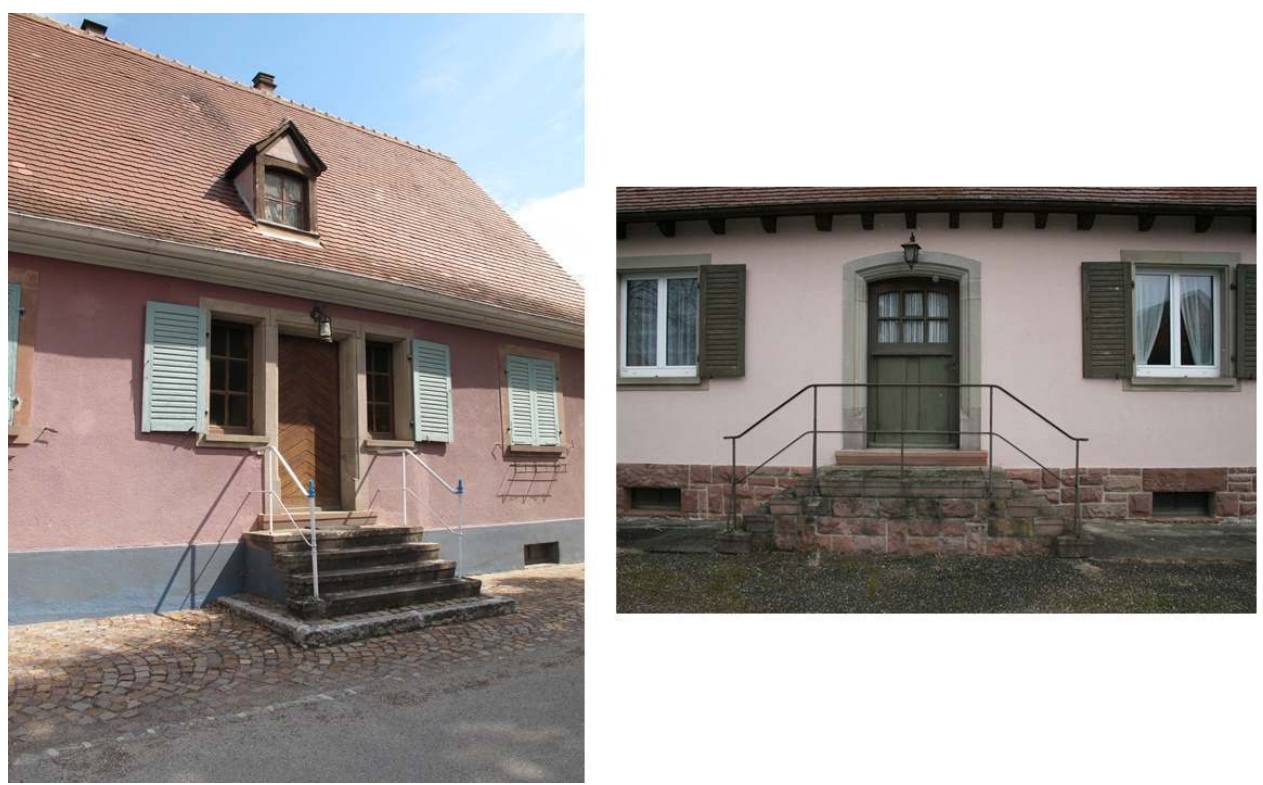

Entrée du logis d'une Erbhof.

Séverine Wodli, 2010. (c) DRAC Alsace.

La fonction agricole de ces fermes prédomine clairement sur celle de logement. Cependant, c'est le logis qui joue un rôle essentiel dans la présentation du statut social de son exploitant. Bien qu'ils soient exactement de même dimension dans les deux cas, une distinction s'opère par leur orientation et par leur décor. L'accès principal du logis d'une Erbhof se fait depuis la rue sur le mur gouttereau qui lui est parallèle, tandis qu'on accède à celui d'une Mittlere Landwirt par la cour. Dans les deux cas, le rez-de-chaussée est surélevé d'un mètre, accessible par quelques marches en grès. Dans le cas d'une Erbhof, le dessin de la travée d'entrée comprend une porte à linteau droit encadrée de deux petites 
fenêtres, ce qui lui confère un caractère plus urbain. Celle d'une Mittlere Landwirt présente un traitement plus rustique.

\section{Aspects architecturaux}

Un procédé unique a été appliqué pour la construction de la Cité paysanne. L'usage du "plan-type » implique une rigidité dans les principes de construction qui se répercute principalement sur le gros-œuvre, tout en permettant des modifications secondaires qui n'entravent pas le fonctionnement général de l'exploitation. Comme pour la construction de la Siedlung, ces travaux ont été essentiellement menés par des personnes natives de Marckolsheim et des prisonniers de guerre polonais. Les matériaux de construction sont modernes et le recours au béton, peu coûteux, solide, facile et rapide à mettre en œuvre, est généralisé. Les fondations d'une épaisseur de $35 \mathrm{~cm}$ ont été coulées sur place. L'étable qui est un espace présentant un fort risque d'incendie du à la méthanisation est rendue plus sûre grâce à la mise en œuvre en béton des dalles du premier étage et du rez-dechaussée, des poteaux structurels. Conformément aux principes hygiénistes déjà évoqués, un système d'évacuation de l'air vicié est installé. L'origine des autres matériaux n'a pu être déterminée que partiellement grâce aux factures conservées dans les archives municipales. C'est le cas de certains éléments, poutres et escaliers, qui portent les initiales $H R$ qui désignent l'entreprise de construction fribourgeoise Hans Rempel. La mise en œuvre réfléchie, l'utilisation de matériaux solides et de bonne qualité, tout comme le surdimensionnement des charpentes témoignent bien de la volonté d'assurer la pérennité de ces fermes.

L'ensemble des murs porteurs est réalisé en maçonnerie de brique pleine. Ils présentent une épaisseur de $40 \mathrm{~cm}$ et se composent d'une double rangée de briques par niveau. Les solins des quatorze fermes sont mis en œuvre sans distinction entre le logis et les dépendances agricoles. Ils sont constitués d'un parement en blocs de grès rose brut de carrière $^{55}$ (finition abrupte et blocs de dimensions variables), excepté pour la ML10 dont les fondations en béton restent apparentes et pour la ML6 dont le grès a une finition bouchardée, particularité qui s'explique sans doute par la présence de la stèle commémorative déjà évoquée. Pour ce qui est du traitement des aspects extérieurs des murs, l'appareillage des briques résulte directement de ce mode de pose. On lit en façade une alternance de lignes de briques en boutisse et de lignes de briques en panneresse. La finition non soignée des joints et le léger décalage entre les encadrements en grès et le nu extérieur laissent supposer que ces murs étaient destinés à être enduits. Pourtant, sur les photographies que nous avons consultées, aucun bâtiment de la Cité paysanne n'était enduit à la fin de la Seconde Guerre mondiale. Les toitures de la maison, de la porcherie et de la grange sont à deux pans à $45^{\circ}$, couvertes de tuiles écailles en terre cuite de couleur rouge, à deux ou trois rainures, en pose double, issues d'une fabrication industrielle. Elles se caractérisent par l'absence de débord au niveau des murs pignons : la tuile, légèrement en saillie, est scellée à la chaux vive (fig. $\left.\mathbf{n}^{\circ} \mathbf{1 9}, \mathbf{n}^{\circ} \mathbf{2 0}\right)$. 
Figure 19
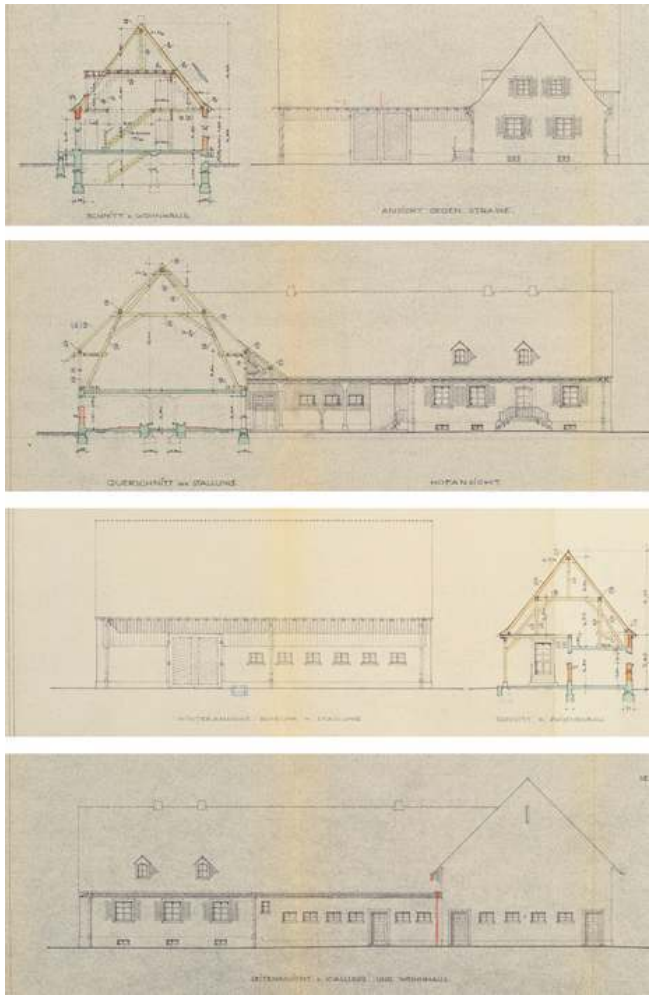

Élévations d'une Mittlere Landwirt, dessinées par le Bureau de la reconstruction de Colmar. 1941. (c) AMM. 
Figure 20

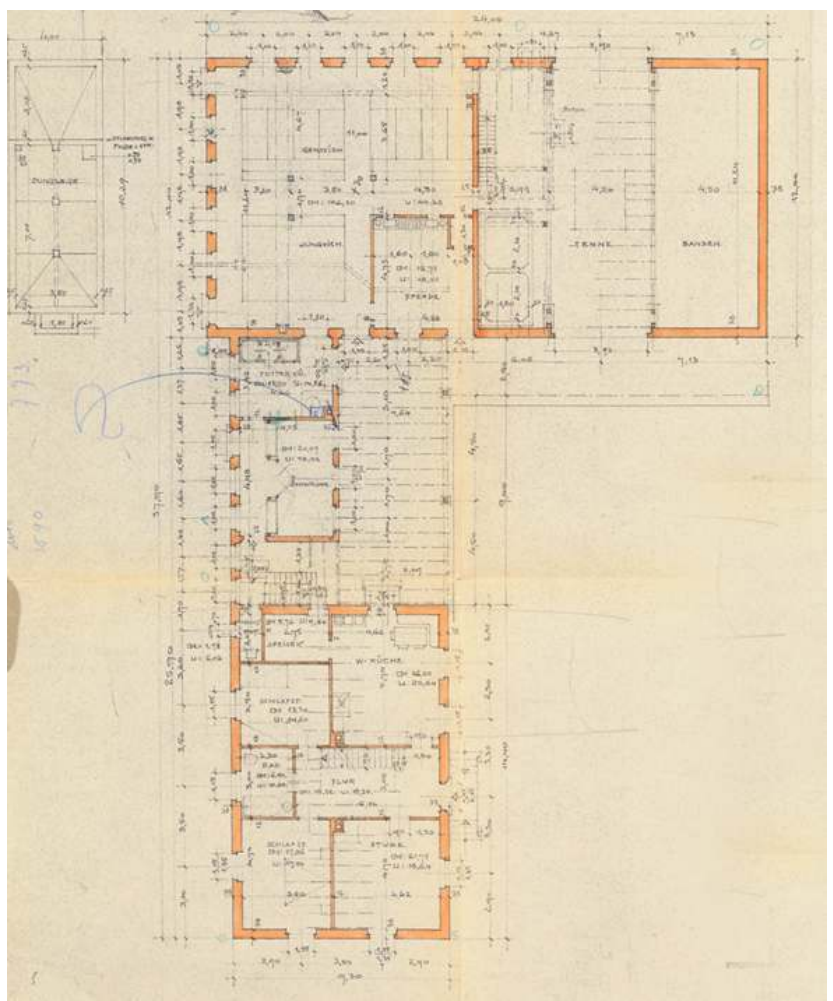

Plans des niveaux d'une Mittlere Landwirt, dessinés par le Bureau de la reconstruction de Colmar. 1941.

(c) AMM.

Différences de taille et d'importance entre les moyennes et les grandes fermes de la Cité paysanne

\begin{tabular}{|l|l|l|}
\hline & Mittlere Landwirt & Erbhof \\
\hline & ML9 $^{56}$ & EB4 $^{57}$ \\
\hline $\begin{array}{l}\text { Surface de terres } \\
\text { cultivées après 1942 }\end{array}$ & 10 ha & 18 ha \\
\hline \multirow{2}{*}{$\begin{array}{l}\text { Surface de la parcelle } \\
\text { de la ferme }\end{array}$} & $1760 \mathrm{~m}^{2}$ & $2554 \mathrm{~m}^{2}$ \\
\hline \multirow{2}{*}{$\begin{array}{l}\text { Surface du logis (sans } \\
\text { la cave) }\end{array}$} & $218 \mathrm{~m}^{2}$ & $218 \mathrm{~m}^{2}$ \\
\hline \multirow{2}{*}{$\begin{array}{l}\text { Surface des } \\
\text { dépendances agricoles }\end{array}$} & $\begin{array}{l}\text { Porcherie }: 21 \mathrm{~m}^{2} \text { (comprenant } \\
\text { box pour les cochons) }\end{array}$ & $\begin{array}{l}\text { Porcherie }: 60 \mathrm{~m}^{2} \text { (comprenant } 4 \\
\text { box pour les cochons) }\end{array}$ \\
\cline { 2 - 4 } & Buanderie $: 16 \mathrm{~m}^{2}$ & Buanderie $: 20 \mathrm{~m}^{2}$ \\
\cline { 2 - 4 } & Fenil :280 $\mathrm{m}^{2}$ & Fenil $: 312 \mathrm{~m}^{2}$ \\
\cline { 2 - 3 } &
\end{tabular}




\begin{tabular}{|l|l|l|}
\cline { 2 - 3 } & Grange $: 134 \mathrm{~m}^{2}$ & Grange $: 150 \mathrm{~m}^{2}$ \\
\cline { 2 - 3 } & Étable $: 126 \mathrm{~m}^{2}$ & Étable $: 142 \mathrm{~m}^{2}$ \\
\cline { 2 - 3 } & Écurie $: 20 \mathrm{~m}^{2}$ & Écurie $: 20 \mathrm{~m}^{2}$ \\
\hline $\begin{array}{l}\text { Nombre de têtes de } \\
\text { gros bétail }\end{array}$ & $\begin{array}{l}6 \text { (mais existence de } 8 \text { box et d'un } \\
\text { box séparé pour le jeune bétail) }\end{array}$ & $\begin{array}{l}6 \text { (mais existence de 15 box et } \\
\text { d'un box séparé pour le jeune } \\
\text { bétail) }\end{array}$ \\
\hline Nombre de chevaux & 2 & 3 \\
\hline $\begin{array}{l}\text { Nombre de têtes de } \\
\text { petit bétail }\end{array}$ & 7 & 9 \\
\hline
\end{tabular}

Le traitement extérieur du logis diffère de celui des dépendances agricoles. Ce principe relevant de l'évidence s'exprime à travers la forme et la dimension des ouvertures : fenêtre rectangulaire verticale pour le logis et fenêtre rectangulaire horizontale pour la dépendance agricole, les types d'encadrement de porte et de fenêtre: encadrement en grès pour le logis et encadrement en briques avec un appui de fenêtre en grès pour la dépendance agricole, et la présence de volets pour les fenêtres du logis. Ces observations sont valables pour les trois modèles de fermes de la Cité paysanne. En revanche, l'analyse des composantes du second œuvre nous révèle que c'est bien à ce niveau que se trouve la plupart des variations d'un type de ferme à l'autre. Outre la différence de traitement de l'entrée principale entre une Erbhof et une Mittlere Landwirt, on constate :

- une variation du nombre d'ouvertures de la grange (18 fenêtres pour les EB contre 13 pour les ML)

- une variation du nombre de lucarnes en toiture du logis (1 pour une EB contre 4 pour une ML)

- un changement d'implantation d'un escalier d'accès au surcroit. Dans le cas de l'EB, la distance entre le logis et la porcherie est plus conséquente que pour une ML. En effet l'escalier, situé perpendiculairement au logis, permet de créer un dégagement accueillant des toilettes extérieures indépendantes, ce qui ajoute un élément de confort non négligeable à l'habitation.

Le logis s'organise sur trois niveaux: la cave semi-enterrée, le rez-de-chaussée, le Ce dernier bénéficie de pièces bien ventilées avec une hauteur importante sous-plafond de $2,70 \mathrm{~m}$. Un couloir central permet de distribuer l'ensemble des pièces principales de la maison : la pièce de vie (Stub), la cuisine, le cellier, la salle de bains et deux chambres à coucher. Il sert de point de transition lors de la venue d'un visiteur entre l'espace privé de la famille et l'espace de réception. Le couloir permet également un accès direct à la cave et à l'étage grâce à deux escaliers droits avec marche et garde-corps en bois. Le départ de celui qui conduit à l'étage est sculpté En plus, des espaces de stockage des pommes de terre et des betteraves, la cave comporte un abri anti-aérien. Les combles se décomposent en quatre pièces : deux chambres et deux pièces servant de grenier. Le plan du rez-dechaussée met en évidence une double hiérarchie des espaces, selon l'axe est-ouest et selon l'axe nord-sud. Les pièces donnant sur la rue sont destinées au chef de famille et 
plus généralement aux hommes, alors que les pièces à l'arrière relèvent davantage du domaine domestique pour la femme et les enfants.

À l'époque de leur livraison, les fermes sont modernes et agréables au regard de l'existant. Les équipements tels que les éviers en grès ou en faïence, les carrelages et les poêles sont livrés avec les habitations. Selon les témoins interrogés, une partir des meubles a été récupérée dans les décombres des bâtiments bombardés en 1940. Les finitions intérieures sont de bonne facture (plancher en bois, évier en grès, garde-corps de l'escalier sculpté, poêle de chauffage à coke) et la construction est raccordée au réseau d'adduction d'eau et d'électricité. L'inventaire révèle des variations d'une ferme à l'autre comme, par exemple, l'installation de deux poêles à coke dans une EB contre un seul dans une ML. Il a permis également de mesurer leur niveau d'intégrité : si elles conservent presque toutes leurs dispositions d'origine, il n'en est pas de même pour leur mobilier.

Le plan des dépendances agricoles témoigne également de la modernité du programme. Le volume de la grange s'organise autour d'une allée centrale permettant de récupérer le foin du fenil par un conduit puis de le distribuer dans les mangeoires des chevaux et du bétail en un seul passage. Outre l'étable et l'écurie, la grange se divise entre les silos en bois pour le stockage des grains, l'aire de battage du blé et le hangar à charrette. Le stockage du foin dans le fenil se faisait à bras d'homme (deux à trois personnes) et à l'aide d'une pince motorisée. On trouve dans la continuité de la porcherie une Futterküche (buanderie ${ }^{58}$ ). Cette pièce est alimentée en eau courante et équipée d'une bouilloire à linge, d'un grand bac en béton et d'un silo en béton partiellement enterré. Un fumoir à viande complète cet ensemble fonctionnel (fig. $\mathbf{n}^{\circ} \mathbf{2 1}, \mathbf{n}^{\circ} \mathbf{2 2}$ ).

Figure 21

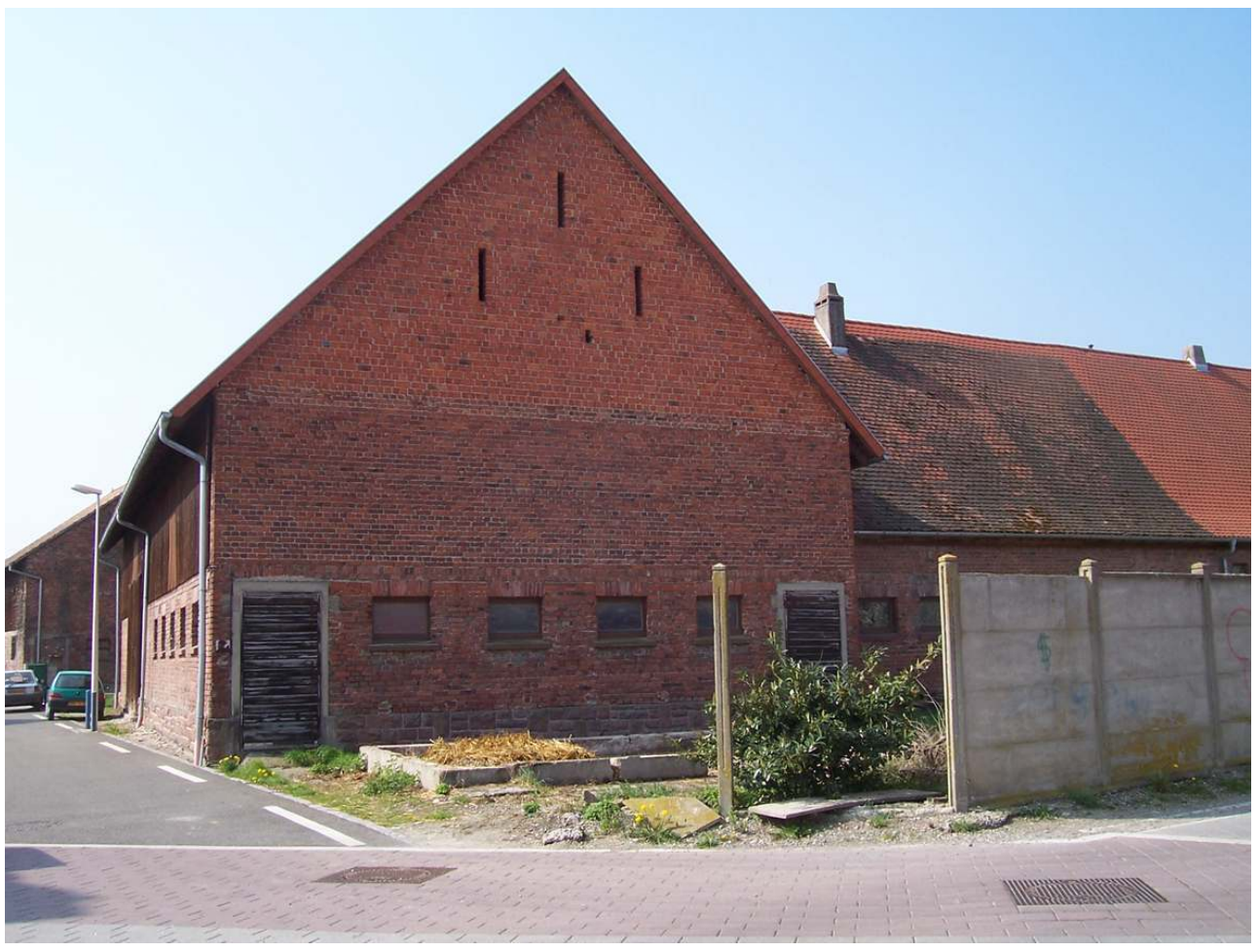

Grange d'une Mittlere Landwirt (ML9) en 2010.

PHOT. SÉVERINE WOdLI. (C) DRAC ALSACE. 


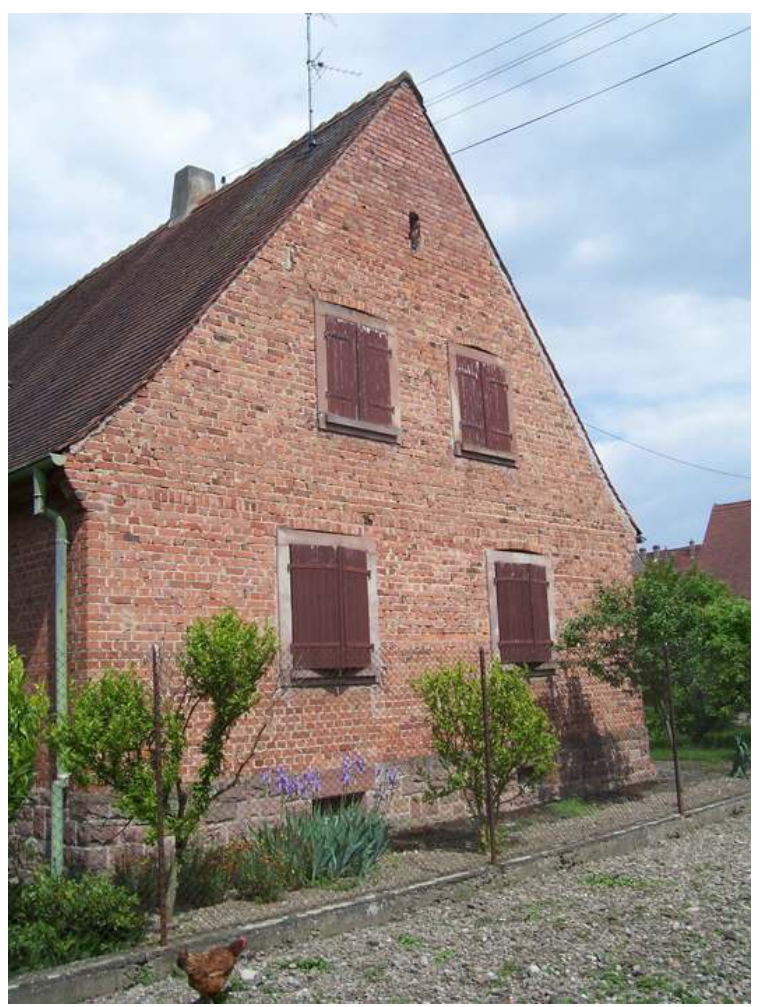

Pignon de la ferme AOLZ3 dans son état d'origine : absence d'enduit, un soubassement en blocs de grès, des tuiles scellées à la chaux sans débord, des encadrements en grès et des volets battants.

PHOT. SÉVERINE WODLI, 2010. @ DRAC ALSACE.

La Cité paysanne de Marckolsheim se maintient aujourd'hui dans ses dispositions d'origine en ce qui concerne son plan général et son inscription paysagère. Le dessin des parcelles actuelles et le réseau viaire ${ }^{59}$ demeure très proche. Les structures et le plan général des fermes sont fidèles au programme de départ. Tous les logis des Erbhöfe et Mittlere Landwirte sont conservés, ainsi que leur cour et l'ensemble des Arbeiter Wohnhaus. Dans le détail, le constat est plus variable. La filiation de ses habitants actuels avec les témoins de sa réalisation est forte, mais la question de l'usage des fermes et de leur transmission ${ }^{60}$ se pose de plus en plus. En ce qui concerne les modifications des dépendances agricoles, outre les ajouts spécifiques déjà présentés (séchoirs à tabac), l'inventaire a identifié la disparition ou la reconstruction de certaines granges suite à des incendies ${ }^{61}$. L'aspect des maçonneries a également évolué puisqu'elles sont enduites presque dans tous les cas ${ }^{62}$ et surtout, elles sont peintes de toutes les couleurs. Les sols des cours ont été parfois pavés ou recouverts de bitume et les clôtures légères remplacées. La pression pour moderniser les installations, notamment de chauffage et d'hygiène, et pour densifier son occupation est néanmoins de plus en plus forte. Certaines dépendances ont déjà été transformées en logement sans véritable prise en compte des qualités patrimoniales de l'édifice individuel et de l'ensemble, mais ce sont encore des cas isolés. Les éléments de second œuvre, extérieurs et intérieurs sont peut-être ceux qui ont le plus souffert du temps, ce qui a nécessité de notre part une observation fine des équipements d'origine encore en place, et la restitution d'un état originel duquel il faudrait se rapprocher dans le cadre de travaux futurs. 


\section{Conclusion}

Le programme de la Cité paysanne semble avoir ses promesses pour les quatorze fermes réalisées. Tous les témoignages recueillis indiquent qu'il est toujours agréable d'y vivre. Ses répercussions sur la vie et le travail, réorganisés au sein de ce quartier en particulier et de la communauté rurale en général, sont positives. Dans un territoire où l'économie rurale est, depuis 1945, de plus en plus tournée vers d'autres activités que l'agriculture, les quatorze fermes composant ce quartier ont globalement conservé leurs caractéristiques paysagères et architecturales. Leur modernité, leur capacité d'adaptation, l'organisation aérée et rationnelle des parcelles et des structures ont permis aux habitants qui le souhaitaient de continuer à cultiver la terre et de faire évoluer leurs moyens techniques. Aujourd'hui, Marckolsheim s'est étendue et la Cité se retrouve au cœur du bourg. Ses habitants y sont attachés et ils se sont approprié son histoire comme son architecture. Tous s'accordent à y voir une part de leur identité ce qui passe par la volonté d'en conserver la cohérence et la qualité.

C'est pourquoi l'étude de la Cité paysanne de Marckolsheim devait être l'occasion d'expérimenter une nouvelle méthode d'inventaire. Sa protection au titre des monuments historiques ${ }^{63}$ devait répondre aux attentes des élus, des propriétaires, des laboratoires de recherche et des institutions patrimoniales. Notre démarche intègre à la fois la recherche historique et des outils d'aide à la décision permettant aux collectivités territoriales de prendre le relais en matière de préservation du patrimoine. La collecte de données relatives aux sciences humaines et sociales lors de l'inventaire offre une vision dynamique du monde rural et de ses mutations. En effet, le bâti rural traditionnel constitue le prolongement du milieu dans lequel il a été créé, ce qui implique qu'il ne doit pas être appréhendé comme un objet unique et isolé mais comme un ensemble à l'échelle territoriale. Par ailleurs, si les précautions nécessaires à la recherche sur cette période douloureuse de l'histoire régionale ont été prises en compte dès le départ, les enjeux de ce travail de recherche se sont très rapidement révélés résider ailleurs. Au-delà des théories constructives et des concepts idéologiques inhérents à ce programme de reconstruction, la Cité paysanne est avant tout l'expression d'un système de production agricole qui renvoie à des problématiques de rentabilité économique, d'amélioration des techniques et d'organisation sociale d'une communauté rurale. Ces perspectives dépassent largement le niveau local de notre étude et en constituent alors l'enjeu majeur.

WODLI, Séverine. Héritages et planification territoriale en milieu rural - Les constructions agricoles en plein cœur du débat, Mémoire de recherche (non publié), École Nationale Supérieure du Paysage de Versailles, 2010, $125 \mathrm{p}$. 


\section{BIBLIOGRAPHIE}

ANONYME. Neue Dorflandschaften,Gedanken und Pläne zum Ländlischen Aufbau in den neuen Ostgebieten und im Altreich. Berlin : Verlag Deutsche Landbuchhandlung Sohnrey \& co, [après 1943], 147 p.

COLLECTIF. Dossier documentaire pour la protection au titre des monuments historiques de la Cité paysanne, 2012. DRAC Alsace, 2010, 300 pages. (non publié)

BOPP, Marie Joseph. L'Alsace sous l'occupation allemande 1940-1945. Le Puy : X. Mappus éditeur, 1945, $374 \mathrm{p}$.

DARRE, R. Walther. Neuadel aus Blut und Boden. München : Éd. J. F. Lehmann, 1930, 231 p.

DENIS, Marie-Noëlle, GROSHENS, Marie-Claude. L'Architecture rurale française - Alsace. Tome 6.

Paris : Berger-Levrault, 1978, 291 p.

KNITTEL, Michel. Marckolsheim - Fragments d'Histoire. Grussheim : Société d'Histoire de la Hardt et du Ried, 1994, 370 p.

MILLIUS, Dominique, FISCHER, Thierry. Connaître et défendre les richesses de notre Ried.

Marckolsheim : Syndicat d'initiative de Marckolsheim, CPIE Régional et Maisons paysannes d'Alsace, 1983, $57 \mathrm{p}$.

SOUBIGOU, Gilles. Le patrimoine des années 1940 en Alsace - rapport d'investigation, Rapport de stage (non publié), promotion Umberto Eco de l'INP, 2008, 48 p.

VIGATO, Jean-Claude. L'architecture régionaliste. France 1890-1950. Paris : Institut Français d'Architecture, Norma éditions, 1994, 390 p.

VOIGT, Wolfgang. Planifier et construire dans les territoires annexés; architectes allemands en Alsace de 1940 à 1944. Collection Recherches et Documents; tome 78, Strasbourg, édition des Publications de la Société Savante d'Alsace, 2008, 215 p.

\section{NOTES}

1. - Avec la contribution d'Olivier HAEGEL, chercheur au Service de l'inventaire du patrimoine de la Région Alsace.

2. - Cette définition est empruntée à la «Convention concernant la protection du patrimoine mondial, culturel et naturel » issue de la conférence générale de l'Organisation des Nations Unies pour l'éducation, la science et la culture, réunie à Paris du 17 octobre au 21 novembre 1972.

3. - Traditionnellement, les historiens de l'architecture considèrent que l'Alsace a connu quatre reconstructions. La première fait suite à l'annexion de l'Alsace en 1871 et concerne avant tout Strasbourg qui, devenant capitale du Reichsland Elsaß-Lothringen connaît une extension urbaine, la Neustadt, comprenant outre des logements, d'importantes institutions: université, hôpital, administrations publiques de la justice, des finances, etc. La deuxième a lieu entre 1918 et 1939, période du retour de l'Alsace à la France, et de la reconstruction des villages détruits lors de la Première Guerre mondiale. La troisième, entre 1940 et 1944, l'Alsace à nouveau annexée fait l'objet de programmes de reconstruction suite aux dommages subis et dans le cadre de 
l'économie de guerre. Et enfin, la quatrième, après 1945, le territoire est reconstruit après la fin de la Seconde Guerre mondiale.

4. - Par exemple, c'est dans ce cadre qu'a été inscrite au titre des Monuments historiques par arrêté du 14 décembre 2009, l'usine List de Rhinau dans le Bas-Rhin. Ce grand bâtiment a été construit en 1942 par Ernst Neufert (1900-1986) ; on y fabriquait des équipements électriques pour les avions de la Luftwaffe et, plus tard pour les fusées V1 et V2. Son intérêt architectural réside, entre autres, dans l'application du système octamétrique) de l'architecte et dans la conception de l'usine comme un module d'une vaste cité industrielle à venir qui devait compter 500 à 700 pavillons de logements.

5. - D'autres localités comme Lauterbourg au nord de la région ont fait l'objet de projets de reconstructions de grande ampleur entre 1940 et 1944. Dans ce cas on conserve une grande partie des archives du bureau de la reconstruction qui a suivi ce projet. Malheureusement, il ne reste plus rien ou presque des réalisations de cette période car la commune a subi de lourds dommages en 1945.

6. - Nous remercions Grégory ZEIGIN, étudiant en ethnologie, stagiaire et troisième auteur du dossier de protection conservé à la DRAC Alsace.

7. - Nous remercions l'ensemble des chercheurs du Service de l'inventaire du patrimoine de la région Alsace, en particulier Jérôme Rimbault. Leur connaissance du terrain et de l'histoire régionale, leurs conseils méthodologiques et leur soutien ont été très souvent d'un grand secours. Nous adressons également nos remerciements amicaux à Sandra Pascalis pour sa lecture avisée et Camille Varin pour la traduction du résumé en anglais.

8. - Le comité de pilotage de l'étude réunit des universitaires dont les enseignants responsables des étudiants accueillis à la DRAC en stage : Rogé Some, professeur d'ethnologie à l'Université de Strasbourg, Benoît Bruant, professeur à l'Université de Haute Alsace, Didier Bouillon, professeur à l'Ecole Nationale Supérieure du Paysage de Versailles, Hervé Doucet, Maître de conférences en histoire de l'art à l'Université de Strasbourg. Nous les remercions pour l'intérêt qu'ils ont porté au sujet, leur disponibilité et leurs précieux conseils. D'autres personnalités encore, comme Wolfgang Voigt, ont participé aux réunions de ce comité et ont très largement contribué à la qualité de ce travail. Enfin, nous remercions les membres du Conseil Municipal de Marckolsheim et en particulier M. Frédéric Pfliegersdoerffer et Mme Claudine Ober, ainsi que les membres de la Commission du patrimoine de la municipalité et M. Régis Adolf qui ont suivi cette démarche de prêt et ont relayé ses enjeux localement.

9. - Selon les recherches de Wolfgang Voigt, l'Alsace compte cinq bureaux de la reconstruction en Alsace : Colmar (dont dépend Marckolsheim), Lauterbourg, Wissembourg, Soultz-sous-Forêts et Haguenau. (Op cit, p. 148).

10. - Les exploitations de taille moyenne de la Cité paysanne sont désignées à la fois comme Mittlere Landwirt et Mittlere Betrieb. Ces deux termes figurent sur les plans conservés aux archives municipales de Marckolsheim. Le terme de Landwirt se rapporte davantage à l'habitant, et celui de Betrieb au bâtiment. Ainsi, nous n'utiliserons dans cet article que le terme Mittlere Landwirt.

11. - L'usage de " plan-type » renvoie à la question de l'élaboration et de la commande des plans qui reste à approfondir. Les archives ne mentionnent que le Bureau de la reconstruction de Colmar. Or, il n'y a pas de mention des auteurs de ces plans. La question de la filiation du projet architectural des exploitations mériterait d'être analysée au regard des archives des ministères centraux du Reich.

12. - Les entretiens ont porté sur les « histoires de vie» des habitants de la Cité paysanne quand ils étaient en mesure de nous apporter des réponses ou de nous montrer des documents concernant la construction de leur quartier. Les entretiens menés ont été semi-dirigés ou dirigés. Ils permettaient entre autres aux habitants de réagir à la présentation d'illustrations ou d'archives collectées.

13. - Certains propriétaires actuels des fermes sont les enfants des primo-occupants. 
14. - Nous remercions l'ensemble des membres du Conseil municipal et la Commission du patrimoine de commune de Marckolsheim présidée par Mme ober pour leur accueil et leur soutien. Par ailleurs, nous remercions M. Baumgarten de nous avoir permis d'accéder à ses archives privées et en particulier ses cartes postales et photographies, M. Fahrner pour son témoignage de directeur de l'école à l'époque, M. Knittel pour les renseignements historiques qu'il nous a fournis. Nous remercions enfin tous les habitants de la Cité paysanne qui ont été les acteurs clefs de cette enquête : leur disponibilité, leur gentillesse et leur intérêt nous ont permis d'entrer dans leur propriété et d'échanger sur leurs souvenirs.

15. - L'Alsace est considérée comme " annexée de fait " par les Allemands sans officialisation. Le Gauleiter de Bade, Robert Wagner, entreprend la planification de la reconstruction pour les deux côtés du Rhin. À cette date, l'annexion n'est pas reconnue par le gouvernement de Vichy.

16. - Strasburger Neueste Nachrichten, 20.10.1940, BNUS.

17. - Extrait d'un document émanant du bureau de reconstruction de Colmar, AMM, 1 W 670.

18. - Neue Dorflandschaften, Gedanken und Pläne zum Ländlischen Aufbau in den neuen Ostgebieten und im Altreich. Berlin : Verlag Deutsche Landbuchhandlung Sohnrey \& co, [après 1943], p. 45.

19. - Les travaux de Walther Darré sont essentiels dans la définition du programme des communes de l'ordre nouveau. En effet, agronome de formation il a exercé un regard critique sur le monde rural allemand et les dysfonctionnements auxquels il est soumis depuis le début du XX siècle, entre autres dans son ouvrage Neuadel aus Blut und Boden. München : Ed. J. F. Lehmann, 1930.

20. - Par décret du 30 juin 1940 du Reichsstatthalter, les dispositions prises pour le Pays de Bade sont étendues à l'Alsace. Robert Wagner cumule alors trois fonctions. Il est Gauleiter, responsable nazi de la circonscription régionale (propre à ce parti) appelée Gau. Il est encore Reichsstatthalter : gouverneur qui dirige le Reichsland, ancienne division administrative du Reich, et à ce titre responsable devant le parlement régional (ce titre est créé en Alsace en 1879). Dans les territoires annexés (Alsace et Moselle) ce gouverneur est nommé Reichsstatthalter. Enfin, il est Chef der Zivilverwaltung, chef de l'administration civile.

21. - Strasburger Neueste Nachrichten, 01.07.1941, BNUS.

22. - Cité par VOIGT, Wolfgang. Planifier et construire dans les territoires annexés; architectes allemands en Alsace de 1940 à 1944. Collection Recherches et Documents, tome 78, Ed. des Publications de la Société Savante d'Alsace, 215, p. 147.

23. - VOIGT, Wolfgang. Planifier et construire dans les territoires annexés; architectes allemands en Alsace de 1940 à 1944. Collection Recherches et Documents, tome 78, Ed. des Publications de la Société Savante d'Alsace, 215, p. 148.

24. - Strasburger Neueste Nachrichten, 01.07.1941, BNUS.

25. - En ce qui concerne la culture du tabac, il est intéressant d'indiquer que nous nous sommes interrogés sur la présence de séchoirs à tabacs dans plusieurs fermes de la Cité paysanne. En effet, aucun d'entre eux n'apparaissait sur les plans d'origine. En réalité, après avoir questionné les habitants, nous avons établi que ces séchoirs avaient été construits, grâce aux dommages de guerre, après 1945.

26. - Système qui permet le partage des parcelles entre chaque héritier, et entrâne un morcellement des terres agricoles.

27. - Neue Dorflandschaften, Gedanken und Pläne zum Ländlischen Aufbau in den neuen Ostgebieten und im Altreich. Berlin : Verlag Deutsche Landbuchhandlung Sohnrey \& co, [après 1943], p. 104-105.

28. - Le vocabulaire employé ici mérite d'être précisé. En effet, le terme d'Erbhof, que l'on traduit en général par "ferme" recouvre plusieurs aspects qui doivent être bien distingués. L'Erbhof, désigne à la fois la ferme et les bâtiments qui la composent, une exploitation agricole de grande taille, qui possède un statut légal spécifique. Statut qui rejaillit en quelque sorte sur son exploitant, le Bauer (fermier), le plaçant au sommet de la hiérarchie villageoise. Ainsi, pour plus 
de clarté, nous parlons d'«exploitation agricole héréditaire» pour désigner les moyens de production et les terres agricoles élevés au rang d'Erbhof, le terme de «ferme » pour parler du bâti en général (en distinguant le logis des dépendances agricoles), et le terme de "fermier » pour désigner le propriétaire d'une « exploitation agricole héréditaire ».

29. - Op. cit. Wolfgang Voigt, p. 147.

30. - Ibid, p. 147.

31. - Ibid, p. 147.

32. - Un foyer est composé d'un chef de foyer (en général le père quand il est vivant), son épouse, et leurs enfants.

33. - La commune de Marckolsheim compte 1213 habitants dont 313 hommes, 316 femmes, 44 veufs, 50 veuves, 23 célibataires, 241 enfants de moins de 14 ans, 153 enfants de plus de 14 ans, 37 enfants de 21 à 25 ans et 153 personnes rattachées à la famille (frères, sœurs, grands parents, oncles et tantes).

34. - Si l'administration allemande a construit six exploitations de type Mittlere Landwirt, ce sont seulement quatre habitants ayant le statut de Mittlerelandwirt qui habitent dans la Cité paysanne. Les deux exploitations restantes sont occupées par deux habitants ayant le statut d'Erbhof.

35. - Le statut des personnes qui ont habité les maisons-blocs de la Cité paysanne, nommées sur les plans AOLZ, n'est pas clairement défini.

36. - Des plans issus des archives municipales de Marckolsheim, datant d'octobre et novembre 1942, confirment l'intervention de l'administration allemande pour la reconstruction partielle d'exploitations agricoles en dehors de la Cité paysanne. Par exemple, la ferme de la Hueb, située à l'ouest du bourg, est bien documentée. Cette exploitation, appartenant initialement à deux frères, ayant chacun obtenu le statut de Bauer, a été divisée en deux et dotée de deux dépendances agricoles supplémentaires. Les plans, coupes et élévations des projets de ces extensions qui répondent aux mêmes exigences que les équipements contemporains de la Cité paysanne sont très précis.

37. - C'est le cas pour le foyer d'Alphons Meyer habitant la ferme ML5, et Georg Riegert habitant la ML10. Dans les deux cas, ces agriculteurs possèdent bien le statut d'Erbhof mais exploitent une ferme de taille moyenne de type ML. Dans les deux cas, cette situation s'explique par le nombre d'enfants en capacité de travailler dans l'exploitation : 4 dont 3 de plus de 14 ans pour Alphons Meyer et 5 donc 4 de moins de 14 ans pour Georg Riegert, et l'âge du chef de foyer : 57 pour Alphons Meyer et 66 pour Georg Riegert.

38. - Dès son arrivée, l'administration allemande désigne le Orstbauernführer, le chef des agriculteurs du Reich, Alfons Wendling, également Erbhof, utilisé comme relais de l'administration auprès des paysans. Il a permis à l'administration de déterminer les statuts des habitants. Il habite l'EB4.

39. - Voir le tableau présentant les caractéristiques de l'ensemble des foyers de Marckolsheim.

40. - Voir note 37.

41. - Strasburger Neueste Nachrichten, 01.07.1941, BNUS.

42. - Strasburger Neueste Nachrichten, 30.06.1941, BNUS.

43. - Strasburger Neueste Nachrichten, 01.07.1941, BNUS.

44. - Ce document datant du 26 juin 1941 présente ainsi le programme : «11h31 - Discours du Gauleiter avec lecture de la copie de l'acte. Immédiatement après le discours du Gauleiter le coffret sera emmuré et la pierre posée, pendant que le chœur des RAD chantera la chanson festive du travail « Dieu bénit le travail ». Le Gauleiter prendra ensuite le marteau. » AMM.

45. - L'extrait du texte contenu dans la stèle cité ici est retranscrit dans l'ouvrage de Michel KNITTEL. Marckolsheim - Fragments d'Histoire. Grussheim : Société d'Histoire de la Hardt et du Ried, 1994, p. 67.

46. - On peut lire dans la presse «Ce sont ces innovations, que nos agriculteurs remercient chaleureusement. » Strasburger Neueste Nachrichten, 01.07.1941 (๔ BNUS). 
47. - La «Fête des charpentes » en automne 1941 qui célèbre une étape importante dans les travaux de réparation des maisons endommagées du bourg est, comme le montre l'iconographie diffusée à cette occasion, tout à fait importante (voir Fig. $\left.\mathrm{n}^{\circ} 10\right)$.

48. - Vogesen Heimat Kalender, 1942. Archives privées.

49. - Le bâti rural traditionnel à Marckolsheim est caractérisé par une pluralité de bâtiments s'articulant autour d'une cour sous la forme d'un L, le logis implanté perpendiculairement en limite parcellaire sur rue et une clôture légère entre l'espace public et l'espace privé. Les constructions étaient réalisées en pans de bois, avec, dans certains cas, un soubassement en grès. Elles présentaient toutes une toiture à deux pans à $45^{\circ}$ en tuiles plates écailles traditionnelles appelées Bieberschwanz. DENIS, Marie-Noëlle, GROSHENS, Marie-Claude. L'Architecture rurale française - Alsace. Tome 6. Paris : Berger-Levrault, 1978.

50. - Le numéro attribué aux bâtiments reprend celui du bureau de la reconstruction de Colmar sur le plan d'aménagement de juillet 1941 servant de référence. Formant la première phase de construction, les exploitations de type Erbhof et Mittlere Landwirt ont été numérotées de façon continue, indépendamment $\mathrm{du}$ statut de l'exploitation et probablement selon l'ordre de construction envisagé. En ce qui concerne les Arbeiter Wohnhaüser, leur numérotation reprenant à 1 laisse supposer qu'elles appartiennent à une seconde phase (voir Fig. $\mathrm{n}^{\circ}{ }^{12}$ ).

51. - Un plan d'état des lieux du centre-bourg de Marckolsheim a été réalisé par l'administration allemande suite aux bombardements de 1940, repérant le bâti détruit et le bâti conservé. L'analyse de ce document suggère deux sites possibles d'implantation du quartier de la Cité paysanne: le premier situé au cœur du centre-bourg et le second correspondant à l'emplacement actuel du quartier.

52. - Un article de presse paru dans les Strassburger Neueste Nachrichten en mai 1943 (๔ BNUS) annonce l'entrée des premiers habitants dans les moyennes et grandes fermes de la Cité.

53. - Les Arbeiter Wohnhaüser sont des maisons-blocs (abritant à surface égale et sous le même toit le logis et une petite dépendance agricole accueillant du bétail) destinées à des ouvriers agricoles exploitant moins de 5 ha de terres.

54. - DENIS, Marie-Noëlle, GROSHENS, Marie-Claude. L'Architecture rurale française - Alsace. Tome 6. Paris : Berger-Levrault, 1978.

55. - La provenance des blocs de grès n'a pas pu être déterminée clairement: selon certains témoignages des habitants de la Cité paysanne que nous avons recueillis, les blocs proviendraient de l'église paroissiale. Cette dernière, endommagée lors des bombardements de 1940, a été dynamitée par l'administration allemande pour laisser l'espace libre afin de redessiner le noyau central de Marckolsheim. S'il peut être réaliste d'utiliser ces blocs de grès endommagés pour remblayer le sol, il est assez improbable que leur qualité après explosion ait permis de les réemployer en parement. Cependant, il est vrai que cet épisode traumatisant tient une place particulièrement importante dans la mémoire collective des habitants.

56. - Statistiques réalisées à partir des données correspondant au foyer de Joseph Koch, Mittlerelandwirt, habitant la ferme ML9, 63 ans, veuf, père de trois enfants de 21 à 25 ans. Ce foyer est pris comme exemple représentatif.

57. - Statistiques réalisées à partir des données correspondant au foyer d'Alfons Wendling, Erbhof , habitant l'EB4, 40 ans, marié à une femme de 42 ans, et père de deux enfants de moins de 14 ans. Ce foyer est pris comme exemple représentatif.

58. - La buanderie est une pièce à usage domestique et à fonctions multiples. Sa caractéristique principale réside dans l'installation d'un foyer en maçonnerie qui sert à la cuisson : eau pour la lessive, alimentation des animaux, alambic, etc. En général, on l'utilise pour les travaux nécessitant de l'espace et salissants.

59. - Les rues ont été viabilisées dans les années 1950 et un aménagement paysager du Stadtgraben a été mis en œuvre dans les années 2000. On note également la privatisation partielle de la rue de la Dordogne : elle a été intégrée à la propriété de l'AOLZ2. 
60. - Au moment de l'étude, quatre exploitations sur les dix fonctionnent encore, mais leurs propriétaires sont obligés d'exercer une autre activité pour compléter leur revenu insuffisant depuis plusieurs années.

61. - ML10 et EB3 - pas toujours dans les volumes et les matériaux d'origine. Le volume de la grange de la ML n¹0 a été transformé en menuiserie dans les années 1950.

62. - L'AOLZ3 et la ML9 sont les seules exploitations dont l'intégralité des façades (logis et dépendances) n'a jamais été enduite. En dehors de ces deux exploitations, toutes les façades des logis ont été enduites. On trouve aujourd'hui encore quelques dépendances agricoles non enduites, mais elles ne représentent pas la majorité (porcherie de l'EB 1, grange et porcherie de l'EB 4, la ML7).

63. - L'arrêté d'inscription de la Cité Paysanne de Marckolsheim est signé le 24 octobre 2012.

\section{RÉSUMÉS}

La Conservation régionale des monuments historiques d'Alsace a engagé depuis 2008 une enquête thématique sur le patrimoine architectural de la reconstruction allemande (1940-1944), qui a débuté par l'étude et la valorisation du quartier de la Cité paysanne de Marckolsheim. Cet ensemble de quatorze «fermes modèles » est remarquable à plusieurs titres : par son exemplarité et la publicité qui en a été faite à l'époque, par la richesse de sa documentation, par sa conservation urbaine et architecturale. Il est le témoin de la volonté politique de transformer profondément la société rurale et l'économie agricole par l'introduction de la modernité et la révision de la hiérarchie sociale. Par ailleurs, du fait de la nature de cet ensemble et de la compréhension du contexte historique qui l'a vu naître, cette étude a permis de réinterroger la notion même de patrimoine, ainsi que la procédure de protection au titre des monuments historiques et ses conséquences réglementaires.

In 2008, the Conservation Régionale des Monuments Historiques d'Alsace (Regional Centre for Heritage Conservation in Alsace) launched a thematic survey on the architectural heritage of reconstruction in Alsace, undertaken by the Germans between 1940 and 1944. It started with the study and interpretation of the Cité Paysanne ("peasant settlement") district. This series of fourteen "ideal farms" is remarkable in several ways: its exemplarity and its publicity at the time, the extent of the documentation concerning it, as well as its urban and architectural preservation. This ensemble bears witness to the political will bring about a radical transformation of rural society and the agricultural economy by introducing modernity and renewing the social hierarchy. Furthermore, in considering the nature of this ensemble and the historical context of its construction, this survey led us to think anew about the notion of cultural heritage as well as the procedures which exist for its conservation as historical monuments.

\section{AUTEURS}

\section{CLÉMENTINE ALBERTONI}

Chargée de la protection des monuments historiques à la DRAC Alsace clementine.albertoni@culture.gouv.fr 


\section{LAURINE SANDOVAL}

Historienne laurine.sandoval@hotmail.fr

\section{SÉVERINE WODLI}

Architecte sev.wodli@gmail.com 